\title{
Sensitivity of spectral lines to temperature, velocity, and magnetic field ${ }^{\star}$
}

\author{
D. Cabrera Solana ${ }^{1}$, L. R. Bellot Rubio ${ }^{1,2}$, and J. C. del Toro Iniesta ${ }^{1}$ \\ ${ }^{1}$ Instituto de Astrofísica de Andalucía (CSIC), Apdo. de Correos 3004, 18080 Granada, Spain \\ e-mail: dcabrera@iaa.es \\ 2 Kiepenheuer-Institut für Sonnenphysik, Schöneckstr. 6, 79104, Freiburg, Germany \\ Received 18 January 2005 / Accepted 1 April 2005
}

\begin{abstract}
We present an analytical and numerical study of the sensitivity of weak solar photospheric lines to temperature, velocity, and magnetic fields. Our investigation is based on the concept of response functions (Landi degl'Innocenti \& Landi degl'Innocenti 1977; Ruiz Cobo \& del Toro Iniesta 1994). Lines commonly used in solar spectropolarimetry, like Fe I $630.25 \mathrm{~nm}$ in the visible and Fe I $1564.85 \mathrm{~nm}$ in the infrared, are examined in detail as emerging from reference quiet Sun and sunspot models. We develop a simple phenomenological model capable of describing the response of any given line to these atmospheric parameters. We find that: (a) the sensitivity of the lines to velocity and magnetic fields increases with the sharpness of the intensity and circular polarization profiles; (b) the sensitivity to temperature is determined mainly by the variation of the source function with temperature, which is smaller at longer wavelengths; and (c) lines quoted to be insensitive to temperature, like Fe I $1564.85 \mathrm{~nm}$ and Fe I $557.61 \mathrm{~nm}$, exhibit larger changes in equivalent width than lines presumed to have higher sensitivities to $T$, such as Fe I $630.25 \mathrm{~nm}$. The relations provided by our model are universal and can be used to decide which line is better suited to measuring a given atmospheric parameter. The results of this study are of practical interest for the design of new instruments and for better exploitation of existing ones.
\end{abstract}

Key words. Sun: photosphere - line: profiles - Sun: magnetic fields - polarization

\section{Introduction}

Astrophysicists obtain information about the thermal, magnetic, and dynamic structure of the solar atmosphere by measuring and interpreting the intensity and polarization profiles of spectral lines. In doing so they exploit the fact that the physical parameters of the atmosphere leave clear signatures in these profiles. For example, mass motions shift the spectral lines, whereas magnetic fields induce circular and linear polarization. Different levels of sophistication can be used to interpret the observations, from simple measurements of line bisectors or equivalent widths to complex inversions of the radiative transfer equation. In all cases, it is agreed that one should carefully select the spectral lines to be observed, so that they react mainly to one atmospheric parameter. By combining lines that are sensitive to different parameters, it is in principle possible to uniquely determine the physical properties of the medium where they are formed.

Unfortunately, such an ideal case is only seldom encountered in practice. Magnetically insensitive lines do exist, but all lines react to temperature and velocities to a larger or smaller extent. If, for example, one is interested in determining the velocity field in a sunspot by means of bisector analyses, it is

\footnotetext{
* Appendix A is only available in electronic form
} http://www.edpsciences.org clear that the appropriate line should be magnetically insensitive. The selected line should also exhibit little response to temperature, but it is hopeless to expect zero temperature sensitivities. The claim that certain lines do not react to temperature has caused some confusion in the literature.

Once these general considerations are agreed upon, the question remains as to how to select the best lines for the particular problem under consideration. Over the years, lines suitable for measuring magnetic fields have been identified on the basis of their large Zeeman splittings (e.g., von Klüber 1948; Solanki et al. 1992; Rüedi et al. 1998) or some other special properties as, for example, the absence of linear polarization (which removes undesired instrumental crosstalk between the Stokes parameters; see Vela Villahoz et al. 1994) and their large Stokes $V$ amplitudes (e.g., Solanki et al. 1987, 1990; Rüedi et al. 1995). Also, lines with zero Landé factors have been identified to allow meaningful Doppler shift measurements in sunspots (e.g., von Klüber 1948). It is important to bear in mind, however, that the sensitivity to magnetic fields is not determined by the amount of Zeeman splitting alone. In fact, lines with similar Zeeman splittings but different thermal widths are seen to exhibit different responses to magnetic fields.

The response of spectral lines to temperature and velocities, by contrast, is not so well characterized. Although simple considerations indicate how a given line reacts to temperature 
(e.g., Gray 1992), these estimates must be viewed with caution because of the many simplifying assumptions on which they are based ${ }^{1}$. To the best of our knowledge, no detailed analysis of the sensitivity of spectral lines to mass motions has ever been published. Thus, one is forced to rely on an intuition that lines in the red part of the spectrum are better suited to the determination of velocities because the Doppler shift is proportional to wavelength.

The importance of a proper selection of spectral lines has been emphasized by recent advances in solar instrumentation. We are now able to put spectrographs and polarimeters in space and to observe the near infrared part of the spectrum from the ground. Prominent examples of existing or future space-based/balloon-borne instruments include the Michelson Doppler Imager (MDI; Scherrer et al. 1995) onboard SOHO, the Solar-B spectropolarimeter (Lites et al. 2001), the vector magnetograph and visible spectropolarimeter of Sunrise (Solanki et al. 2003; Gandorfer et al. 2004), the Helioseismic and Magnetic Imager (HMI, Scherrer 2002) onboard the Solar Dynamics Observatory, and the Visible-light Imager and Magnetograph of Solar Orbiter (Marsch et al. 2002; see also the proceedings edited by Battrick \& Sawaya-Lacoste 2001). The lines observed by these instruments are often picked up from a small list of candidates for which detailed radiative transfer calculations are carried out. This limited search range is imposed, among other reasons, by the lack of a simple formulation capable of describing the sensitivity of the lines to the various atmospheric parameters.

In this paper we study the sensitivity of spectral lines to the physical conditions of the solar atmosphere, that is, to temperature, velocity, and magnetic fields. We follow two complementary approaches. First, we compute response functions for a set of lines widely used in solar physics. Both visible and near infrared lines are considered. We investigate the sensitivity of these lines in three different model atmospheres simulating the conditions of sunspot umbrae and penumbrae, as well as the quiet sun. Second, we develop an analytical model that is able to explain the sensitivities of the lines as inferred from the numerical calculations. This model provides relations that can be used to determine the sensitivity of any line by plugging in simple parameters, such as line widths and residual intensities.

To illustrate the diagnostic potential of visible and infrared lines, we selected the Fe I $630.25 \mathrm{~nm}$ and Fe I $1564.85 \mathrm{~nm}$ lines for closer scrutiny. These are the lines measured by a number of state-of-the-art spectropolarimeters, including the Advanced Stokes Polarimeter (ASP, Elmore et al. 1992), the Polarimetric Littrow Spectrograph (POLIS, Schmidt et al. 2003), the La Palma Stokes Polarimeter (LPSP, Martínez Pillet et al. 1999), and the Tenerife Infrared Polarimeter (TIP; Collados et al. 1999).

The paper is organized as follows. The meaning of response functions, details of the numerical calculations, and the description of our analytical model are given in Sect. 2. We investigate the sensitivity of the selected lines to velocities, magnetic fields, and temperatures in Sects. 3-5, respectively.

\footnotetext{
1 The most important is perhaps the neglect of variations in the source function when the temperature is changed.
}

A discussion of the results is given in Sect. 6. Finally, Sect. 7 summarizes our conclusions.

\section{Sensitivity of spectral lines}

According to Ruiz Cobo \& del Toro Iniesta (1994), the sensitivity of Stokes profiles to perturbations of the atmospheric parameters are given by the so-called response functions (RFs). They provide direct information on how changes in the physical conditions of the solar atmosphere cause modifications of the emergent spectrum. Response functions appear naturally after linearization of the radiative transfer equation, and were first called weight functions by Mein (1971); RFs were extended to polarized radiative transfer by Landi degl'Innocenti \& Landi degl'Innocenti (1977).

Let us summarize here the definition and main properties of RFs. Following del Toro Iniesta (2003), we shall call $x_{i}\left(\tau_{\mathrm{c}}\right)$ a generic atmospheric parameter (index $i$ will denote temperature, magnetic field strength, inclination or azimuth, line-ofsight velocity, etc.) as a function of the continuum optical depth at $500 \mathrm{~nm}, \tau_{\mathrm{c}}$. Modification of the observed Stokes spectrum ${ }^{2}$, $\delta \boldsymbol{I}\left(\tau_{\mathrm{c}}=0 ; \lambda\right)$, after small perturbations $\delta x_{i}\left(\tau_{\mathrm{c}}\right)$ is given by

$\delta \boldsymbol{I}(0)=\sum_{i=1}^{m} \int_{0}^{\infty} \boldsymbol{R}_{i}\left(\tau_{\mathrm{c}}\right) \delta x_{i}\left(\tau_{\mathrm{c}}\right) \mathrm{d} \tau_{\mathrm{c}}$

where $m$ stands for the number of physical quantities relevant to line formation, and the response functions can be written as

$\boldsymbol{R}_{i}\left(\tau_{\mathrm{c}}\right) \equiv \boldsymbol{O}\left(0, \tau_{\mathrm{c}}\right)\left[\boldsymbol{K}\left(\tau_{\mathrm{c}}\right) \frac{\partial \boldsymbol{S}}{\partial x_{i}}-\frac{\partial \boldsymbol{K}}{\partial x_{i}}\left[\boldsymbol{I}\left(\tau_{\mathrm{c}}\right)-\boldsymbol{S}\left(\tau_{\mathrm{c}}\right)\right]\right]$,

with $\boldsymbol{O}\left(0, \tau_{\mathrm{c}}\right)$ the evolution operator from $\tau_{\mathrm{c}}$ to the surface, $\boldsymbol{K}$ the propagation (or absorption) matrix normalized to the continuum absorption coefficient at $500 \mathrm{~nm}$, and $\boldsymbol{S}$ the source function vector. Equation (2) reflects the fact that perturbations $\delta x_{i}$ induce modifications in both $\boldsymbol{K}$ and $\boldsymbol{S}$. These modifications evolve through the atmosphere, as governed by the evolution operator, until they reach the observer. Equation (1) draws a complicated scenario, in which the sometimes subtle differences between the Stokes profiles of a single line as formed in various solar atmospheres are made up of a sum of contributions, one for each of the many atmospheric quantities. The sensitivity of the Stokes vector to perturbations of each quantity is given by the corresponding response function vector: $\boldsymbol{R}_{\boldsymbol{i}}$ tells us how much $\delta x_{i}$ "contributes" to $\delta \boldsymbol{I}(0 ; \lambda)$.

\subsection{Sensitivity to constant perturbations}

RFs have proven useful in practical applications. For example, they are incorporated in a number of inversion codes including SIR (Stokes Inversion based on Response functions, Ruiz Cobo \& del Toro Iniesta 1992) and SPINOR (Frutiger 2000). Unfortunately, the quantitative interpretation of the RFs

\footnotetext{
2 As usual, by Stokes spectrum we understand the four Stokes parameters as functions of wavelength, $\lambda$. We will formally represent it as a vector $I(0 ; \lambda) \equiv(I, Q, U, V)$. The wavelength dependence is dropped in Eq. (1) for simplicity.
} 
is not straightforward. There is, however, a particularly interesting case in which the meaning of RFs can easily be understood. Assume that we are just dealing with constant perturbations, i.e.,

$\delta x_{i}\left(\tau_{\mathrm{c}}\right)=\delta x_{i}=$ const.

$$
\forall i=1,2, \ldots, m \text {. }
$$

In such a case, Eq. (1) reduces to

$\delta \boldsymbol{I}(0)=\sum_{i=1}^{m} \boldsymbol{R}_{i}^{\prime} \delta x_{i}$,

where

$\boldsymbol{R}_{i}^{\prime} \equiv \int_{0}^{\infty} \boldsymbol{R}_{i}\left(\tau_{\mathrm{c}}\right) \mathrm{d} \tau_{\mathrm{c}}=\frac{\partial \boldsymbol{I}(0)}{\partial x_{i}}$.

Therefore, the integrated response functions $\boldsymbol{R}_{i}^{\prime}$ play the role of partial derivatives of the emergent Stokes spectrum with respect to a given atmospheric parameter. Since the concept of partial derivative is familiar to the reader, the usefulness of RFs is quickly understood.

\subsection{Calculation of RFs}

In the following sections, the sensitivity of visible and infrared lines to various atmospheric parameters is characterized by their RFs as evaluated in three solar model atmospheres: the Harvard-Smithsonian Reference Atmosphere of the quiet sun (HSRA, Gingerich et al. 1971), the penumbral model of del Toro Iniesta et al. (1994), and the hot umbral model of Collados et al. (1994). The lines selected for analysis are listed in Table 1 together with their atomic parameters. All of them are widely used in solar physics. Our list contains some magnetically insensitive transitions (with zero effective Landé factor, $\left.g_{\text {eff }}\right)$ and lines that are often quoted to be insensitive to temperature, such as Fe I $557.61 \mathrm{~nm}$.

The RFs as a function of wavelength and optical depth (Eq. (2)) are computed numerically using the SIR code. In order to illustrate the differences between visible and infrared lines, the RFs will be discussed in detail for Fe I $630.25 \mathrm{~nm}$ and Fe I $1564.85 \mathrm{~nm}$. Both of them are normal Zeeman triplets with large Landé factors (2.5 and 3.0, respectively), and can be considered as prototypes of visible and infrared lines. The range of optical depths where Fe I $630.25 \mathrm{~nm}$ and Fe I $1564.85 \mathrm{~nm}$ are formed is determined by the wavelength of the transition, the excitation potential of the lower atomic level, and the oscillator strength. In general, the Fe I line at $1564.85 \mathrm{~nm}$ is able to probe deeper photospheric layers because of the reduced continuum opacity of $\mathrm{H}^{-}$in the infrared (the minimum opacity occurs at $1642 \mathrm{~nm}$ ). Fe I $1564.85 \mathrm{~nm}$ is not formed in high photospheric layers due to its large excitation potential: such layers are cool and the number of atoms capable of absorbing goes to zero very quickly. By contrast, the visible Fe I line at $630.25 \mathrm{~nm}$ does not reach layers much lower than $\tau_{\mathrm{c}}=1$ (due to the increased $\mathrm{H}^{-}$opacity), but its smaller excitation potential means that it can be formed in the upper photosphere. These considerations will help us understand some of the differences between the RFs of visible and infrared lines.
Table 1. Set of visible and infrared lines considered in this work. $\lambda_{0}$ represents the central wavelength, $\chi$ the excitation potential of the lower level, $\log g f$ the logarithm of the oscillator strength times the multiplicity of the lower level, and $g_{\text {eff }}$ the effective Landé factor.

\begin{tabular}{lrrrr}
\hline \hline Species & $\lambda_{0}[\mathrm{~nm}]$ & $\chi[\mathrm{eV}]$ & $\log g f$ & $g_{\text {eff }}$ \\
\hline Ni I & 491.20 & 3.75 & -0.87 & 0.00 \\
Fe I & 524.71 & 0.09 & -4.95 & 2.00 \\
Fe I & 525.02 & 0.12 & -4.94 & 3.00 \\
Fe I & 537.96 & 3.69 & -1.65 & 1.00 \\
Ti II & 538.10 & 1.57 & -2.05 & 0.90 \\
Fe I & 557.61 & 3.43 & -0.91 & 0.00 \\
Fe I & 569.15 & 4.30 & -1.36 & 0.00 \\
Fe II & 614.93 & 3.89 & -2.70 & 1.33 \\
Fe I & 617.33 & 2.22 & -2.98 & 2.50 \\
Fe I & 630.25 & 3.69 & -1.24 & 2.50 \\
Fe I & 630.35 & 4.32 & -2.55 & 1.50 \\
Ti I & 630.38 & 1.45 & -1.44 & 0.92 \\
Ni I & 676.78 & 1.83 & -1.89 & 1.50 \\
Fe I & 709.04 & 4.23 & -1.21 & 0.00 \\
Fe II & 722.45 & 3.89 & -3.39 & 0.00 \\
Si I & 1062.76 & 5.86 & -0.29 & 1.75 \\
Fe I & 1089.63 & 3.07 & -2.85 & 1.50 \\
Fe I & 1142.23 & 2.20 & -2.89 & 1.98 \\
Fe I & 1221.33 & 4.64 & -1.93 & 2.50 \\
Fe I & 1558.83 & 6.37 & +0.25 & 1.50 \\
Fe I & 1564.85 & 5.43 & -0.67 & 3.00 \\
\hline
\end{tabular}

For the other lines in Table 1, our sensitivity analysis is based on integrated RFs as defined by Eq. (5). For each line, we integrate over optical depth the RFs provided by SIR, and select the maximum value across the line profile. That is, the sensitivity of a given line will be characterized by a single number to allow easy intercomparisons.

It is important to mention that the Stokes profiles and RFs computed by SIR are normalized to the continuum intensity of the quiet sun (represented by the HSRA model) at the central wavelength of the line. This implies that the Stokes profiles are non-dimensional, and that the units of the RFs are the inverse of those of the corresponding atmospheric parameter. Throughout the paper, the RFs at $\tau_{\mathrm{c}}$ are multiplied by the optical thickness $\Delta \tau_{\mathrm{c}}$ of the corresponding atmospheric layer (the stepsize of the spatial grid used in the calculations is $\Delta \log \tau_{\mathrm{c}}=0.1$ ).

\subsection{Weak line model}

To understand the sensitivity of the lines as inferred from the RFs, we use a simple phenomenological model for the shape of weak spectral lines ${ }^{3}$. More specifically, we assume that the emergent Stokes $I$ profile can be described (in the absence of strong magnetic fields) by a Gaussian of the form

$$
I(\lambda)=A_{2}-A_{0} \exp \left[\frac{-\left(\lambda-\lambda_{0}\right)^{2}}{2 A_{1}^{2}}\right] .
$$

Here, $\lambda_{0}$ stands for the wavelength of the transition, $A_{0}$ and $A_{1}$ are the depth and width of the line, respectively, and $A_{2}$ measures the local continuum intensity.

\footnotetext{
3 The majority of photospheric lines in the solar spectrum are weak.
} 

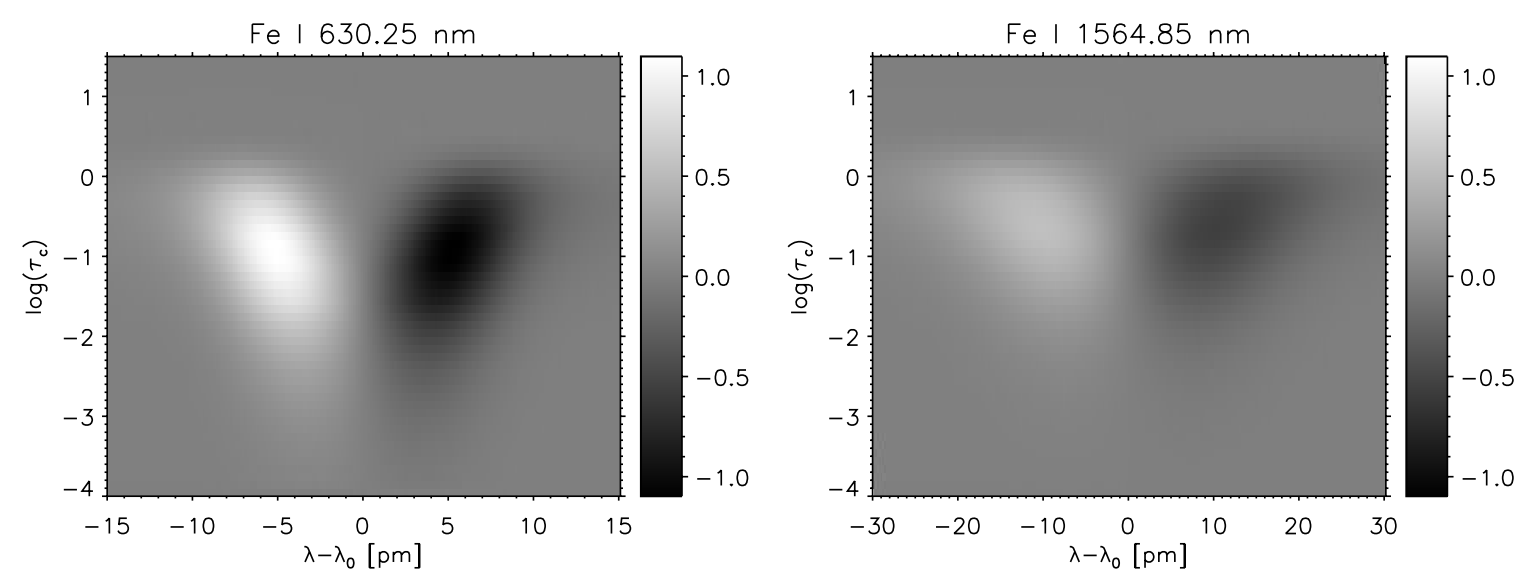

Fig. 1. RF of Stokes $I$ to $v_{\text {LOS }}$ multiplied by $\Delta \tau_{\mathrm{c}}\left[10^{-2}\left(\mathrm{~km} \mathrm{~s}^{-1}\right)^{-1}\right]$ for Fe I $630.25 \mathrm{~nm}$ (left) and Fe I $1564.85 \mathrm{~nm}$ (right) in the HSRA quiet Sun model. The gray scale is the same in the two panels to facilitate comparisons.

In the strong field regime, the $\sigma$ components of the Stokes $V$ profile will be represented as

$V(\lambda)= \pm A_{0}^{V} \exp \left[\frac{-\left(\lambda-\lambda_{0} \pm \lambda_{\mathrm{B}}\right)^{2}}{2\left(A_{1}^{V}\right)^{2}}\right]$

where $A_{0}^{V}$ and $A_{1}^{V}$ are the amplitude and width of the Stokes $V$ lobes, and the Zeeman splitting is $\lambda_{\mathrm{B}}=C g_{\mathrm{eff}} B \lambda_{0}^{2}$, with $C \equiv 4.67 \times 10^{-13} \AA^{-1} \mathrm{G}^{-1}$ and $B$ the field strength (assumed to be constant with height).

For sufficiently small fields, we use the weak field approximation (Landi degl'Innocenti 1992) to represent the Stokes $V$ profile:

$V(\lambda)=-\lambda_{\mathrm{B}} \cos \gamma \frac{\partial I_{0}}{\partial \lambda}$.

Here, $I_{0}$ is the intensity profile that would emerge from the same atmosphere without magnetic fields and $\gamma$ the (heightindependent) inclination of the magnetic field vector. In practice, one observes emergent profiles from the solar atmosphere and they are affected by the magnetic field. For this reason, instead of $I_{0}$ we consider the intensity profile formed in the magnetic atmosphere. If the field is weak enough, this profile has approximately the same shape as $I_{0}$ and can be described by Eq. (6). So inserting Eqs. (6) into (8) leads to

$V(\lambda) \simeq-\lambda_{\mathrm{B}} \cos \gamma A_{0}^{*} \frac{\left(\lambda-\lambda_{0}\right)}{\left(A_{1}^{*}\right)^{2}} \exp \left[\frac{-\left(\lambda-\lambda_{0}\right)^{2}}{2\left(A_{1}^{*}\right)^{2}}\right]$,

where $A_{0}^{*}$ and $A_{1}^{*}$ stand for the depth and width of the intensity profile formed in the atmosphere with magnetic field.

At this point, we emphasize that our description of the Stokes profile shapes does not pretend to be accurate. It just provides simple analytical expressions that can easily be differenciated with respect to the atmospheric parameters.

\section{Sensitivity to line-of-sight velocity}

Figure 1 shows the RFs of Stokes $I$ to velocity as a function of $\lambda$ and $\tau_{\mathrm{c}}$ for Fe I $630.25 \mathrm{~nm}$ and Fe I $1564.85 \mathrm{~nm}$. The HSRA quiet sun model has been used for the computations. No matter the optical depth we are looking at, in the absence of magnetic fields the RFs to LOS velocity perturbations have always two lobes. Since the lines are in absorption, a positive (redshifted) velocity increases the intensities in the blue wing and decreases them in the red wing. For this reason, the RFs show positive blue lobes and negative red lobes. Figure 1 also demonstrates that the visible and infrared lines are sensitive to LOS velocity perturbations in a broad range of layers. That is, the lines are not "formed" at a single depth. Other important properties of the RFs displayed in Fig. 1 are: (a) Fe I $1564.85 \mathrm{~nm}$ is not sensitive to velocities in layers below $\log \tau_{\mathrm{c}} \sim 0$ and above $\log \tau_{\mathrm{c}} \sim-2$; and (b) the visible line shows a greater sensitivity than the infrared line.

Some of these properties are easy to understand. The fact that Fe I $1564.85 \mathrm{~nm}$ does not probe high photospheric layers is due to its large excitation potential, as explained in Sect. 2.2. The other two properties are counter-intuition. Due to the small $\mathrm{H}^{-}$opacity in the infrared, Fe I $1564.85 \mathrm{~nm}$ should react to velocity perturbations in layers deeper than $\log \tau_{\mathfrak{c}} \sim 0$, but this is not what we see in Fig. 1. Also, one would expect larger sensitivities for the infrared line because the Doppler shift is proportional to wavelength. As shown below, the behavior exhibited by the RFs of Fig. 1 can be explained by the phenomenological model introduced in Sect. 2.3.

If the line is formed in an atmosphere in which matter moves at a constant velocity with component along the line of sight $v_{\mathrm{LOS}}$, then the intensity profile of Eq. (6) is shifted by

$\lambda_{\mathrm{LOS}}=\lambda_{0} \frac{v_{\mathrm{LOS}}}{c}$,

and becomes

$I(\lambda)=A_{2}-A_{0} \exp \left[\frac{-\left(\lambda-\lambda_{0}-\lambda_{\mathrm{LOS}}\right)^{2}}{2 A_{1}^{2}}\right]$.

According to the results of Sect. 2.1, the RF of Stokes $I$ to constant perturbations in $v_{\mathrm{LOS}}$ is given by

$$
\begin{aligned}
R_{v_{\mathrm{LOS},}, 1}^{\prime}(\lambda) \equiv \frac{\partial I}{\partial v_{\mathrm{LOS}}}= & -\frac{A_{0} \lambda_{0}}{A_{1}^{2} \mathrm{c}}\left(\lambda-\lambda_{0}-\lambda_{\mathrm{LOS}}\right) \\
& \times \exp \left[\frac{-\left(\lambda-\lambda_{0}-\lambda_{\mathrm{LOS}}\right)^{2}}{2 A_{1}^{2}}\right],
\end{aligned}
$$




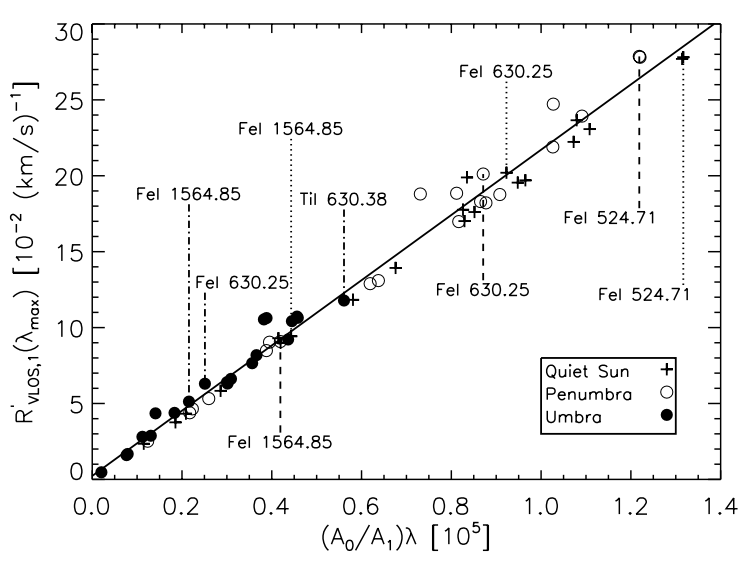

Fig. 2. Maximum value of the integrated RF to $v_{\mathrm{LOS}}$ for the lines of Table 1, as a function of the shape ratio multiplied by the central wavelength of the transition. Quiet Sun (crosses), penumbral (circles) and hot umbral (filled circles) atmospheres have been used. The most sensitive lines, Fe I $630.25 \mathrm{~nm}$, and Fe I $1564.85 \mathrm{~nm}$ are marked with labels in the quiet Sun (dotted lines), penumbral (dashed lines), and umbral (dotted-dashed lines) models.

which has extrema at

$\lambda_{\mathrm{ext}}=\lambda_{0}+\lambda_{\mathrm{LOS}} \pm A_{1}$.

Thus, the maximum sensitivity of the line to velocity shifts is reached in the line wings. Taking the negative sign in Eq. (13), the maximum of the RF is

$R_{v_{\mathrm{LOS}}, 1}^{\prime}\left(\lambda_{\max }\right)=\mathrm{e}^{-1 / 2} \frac{A_{0}}{A_{1}} \frac{\lambda_{0}}{c}$.

Therefore, as one would expect from the Doppler shift relationship in Eq. (10), the larger the wavelength the greater the sensitivity of the line to LOS velocities. But this is only so, however, provided the shape ratio $A_{0} / A_{1}$ remains constant. $A_{0} / A_{1}$ is in fact the ratio between the depth and the width of the line, and can be regarded as a measure of the slope of the intensity profile in the line wings. As mentioned before, Fe I $630.25 \mathrm{~nm}$ shows larger LOS velocity sensitivities than Fe I $1564.85 \mathrm{~nm}$ all across the line profile. A larger shape ratio is the reason for its greater sensitivity. Thus, the shape ratio turns out to be dominant for the line behavior: the stronger and narrower the line, the greater the sensitivity to velocities. Our analytical model also explains why the infrared line is not sensitive to velocities below $\log \tau_{\mathrm{c}} \sim 0$ : near the continuum, the spectral line is so broad that the shape ratio (i.e., the slope of the intensity profile at that position) is almost zero, resulting in very small RFs to $v_{\mathrm{LOS}}$.

Figure 2 shows the maximum of the integrated RF to velocity perturbations for the various lines of Table 1 in the quiet sun, umbral, and penumbral atmospheres. For simplicity, the magnetic field has been set to zero in the umbral and penumbral models ${ }^{4}$. Motivated by Eq. (14), the sensitivity of the lines is plotted as a function of the shape ratio times the central wavelength. Our simple analytical calculations provide an excellent description of the sensitivity

\footnotetext{
${ }^{4}$ In the presence of magnetic fields, the response of magneticallysensitive lines to velocity perturbations would be smaller due to the reduced shape ratios that would result.
}

of visible and infrared lines in the three atmospheres. This has important practical consequences. The relationship shown in Fig. 2 is universal for weak lines: if one is interested in knowing how much a given line reacts to velocity changes, a simple estimate of the coefficients $A_{0}$ and $A_{1}$ (as defined by Eq. (6)) will make it possible to compare its sensitivity with those of other lines. To facilitate such a comparison, Tables A.1-A.3 list the coefficients required to compute the $x$-position of the various lines displayed in Fig. 2.

As can be seen in Fig. 2, the sensitivity of the lines to velocities is generally reduced in the umbral model (filled circles). The reason is the lower temperatures of the umbra as compared with the penumbra and the quiet sun. Such low temperatures produce smaller $A_{0}$ and larger $A_{1}$ (i.e., smaller shape ratios) for the majority of neutral lines in the sample.

In Fig. 2, the most sensitive lines, Fe I $630.25 \mathrm{~nm}$, and Fe I $1564.85 \mathrm{~nm}$ are marked with labels for the quiet Sun (dotted lines), the penumbral (dashed lines), and umbral (dashdotted lines) models. The most sensitive line in the umbra is Ti I $630.38 \mathrm{~nm}$. In the penumbra and the quiet Sun, one should prefer Fe I $524.71 \mathrm{~nm}$ due to its large sensitivity to $v_{\text {LOS }}$. Consistent with our previous findings, the Fe I line at $630.25 \mathrm{~nm}$ is observed to be more sensitive to velocity perturbations than Fe I $1564.85 \mathrm{~nm}$ in all three models.

\section{Sensitivity to magnetic field strength}

In this section, the sensitivity of spectral lines to changes in the magnetic field strength is examined using the RFs for Stokes $V$. We concentrate on the behavior of Stokes $V$ because the determination of magnetic fields in the solar atmosphere relies very often on the interpretation of circular polarization profiles. Stokes $V$ reacts differently to magnetic fields in the strong and weak field regimes, so we need to consider the two cases separately.

\subsection{Strong field regime}

In Fig. 3, the RFs of Stokes $V$ to magnetic field strengths are plotted as functions of $\lambda$ and $\tau_{\mathrm{c}}$ for the Fe I lines at $630.25 \mathrm{~nm}$ and $1564.85 \mathrm{~nm}$. We have used the HSRA quiet sun model with a constant longitudinal $(\gamma=0)$ magnetic field of $B=2000 \mathrm{G}$. The field is sufficiently large so as to consider that the strong field regime applies. The RFs to $B$-perturbations exhibit four lobes, two for each $\sigma$ component. The signs of the RFs reflect how the Stokes $V$ signal varies when the field is increased: in the strong field regime, the amplitudes of the Stokes $V$ lobes do not change, only their wavelength separation does. The main properties of the RFs displayed in Fig. 3 are:

1. The two lines react to $B$ in a broad range of optical depths, from $\log \tau_{\mathrm{c}} \sim 0.2$ to $\sim-3$ in the case of the visible line and from $\sim 0.5$ to $\sim-2.5$ in the case of the infrared line. Thus, $\mathrm{Fe}$ I $1564.85 \mathrm{~nm}$ is able to probe slightly deeper layers than Fe I $630.25 \mathrm{~nm}$.

2. The infrared line is more sensitive to $B$. This is what one would expect from the fact that the Zeeman splitting is proportional to $g_{\mathrm{eff}} \lambda_{0}^{2}$. 

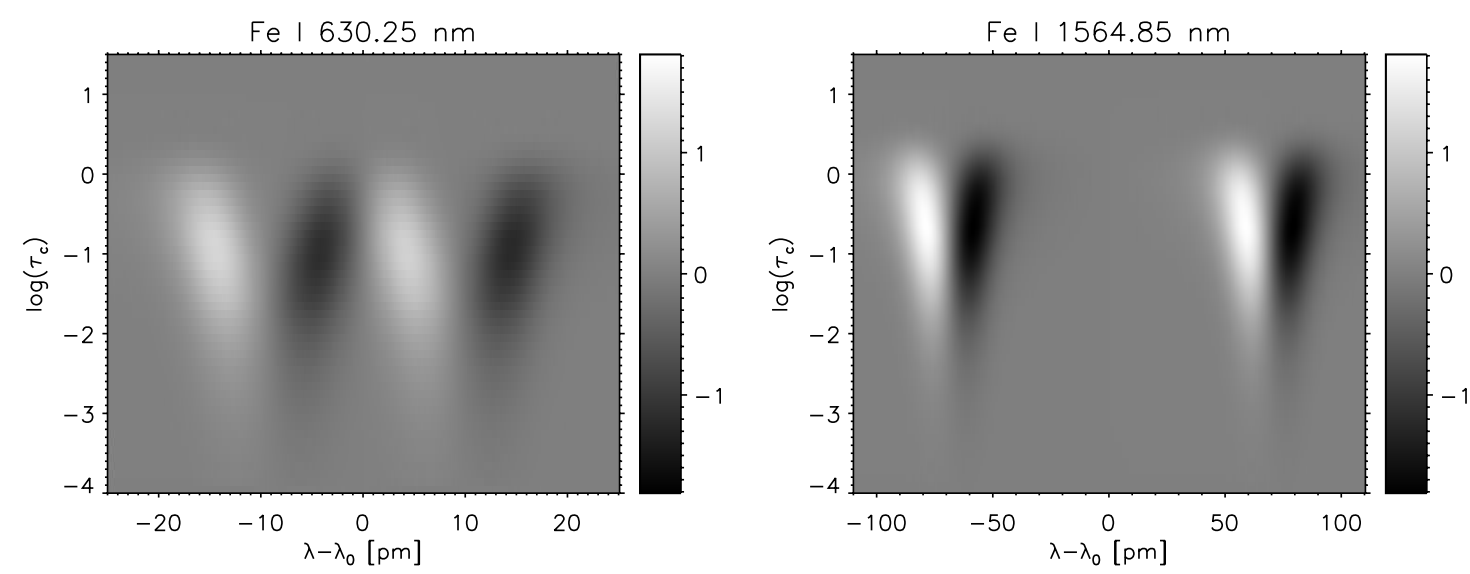

Fig. 3. Normalized RF of Stokes $V$ to $B$ multiplied by $\Delta \tau_{\mathrm{c}}\left[10^{-5} \mathrm{G}^{-1}\right]$ for Fe $\mathrm{I} 630.25 \mathrm{~nm}$ (left) and Fe I $1564.85 \mathrm{~nm}$ (right) in the HSRA model with a longitudinal magnetic field of $B=2000 \mathrm{G}$. The same gray scale is used in the two panels.

We now use the phenomenological model of Sect. 2.3 to gain a better understanding of the line parameters that determine the sensitivity to $B$. In the strong field regime, the lobes of Stokes $V$ can be represented ${ }^{5}$ by Eq. (7). Differenciating this expression with respect to $B$ one obtains that the RF of Stokes $V$ to constant perturbations in $B$ is

$$
\begin{aligned}
R_{B, 4}^{\prime}(\lambda)= & -\frac{A_{0}^{V} C g_{\mathrm{eff}} \lambda_{0}^{2}}{\left(A_{1}^{V}\right)^{2}}\left(\lambda-\lambda_{0}+\lambda_{\mathrm{B}}\right) \\
& \times \exp \left[\frac{-\left(\lambda-\lambda_{0}+\lambda_{\mathrm{B}}\right)^{2}}{2\left(A_{1}^{V}\right)^{2}}\right] .
\end{aligned}
$$

The RF has extrema at

$\lambda_{\text {ext }}=\lambda_{0}-\lambda_{\mathrm{B}} \pm A_{1}^{V}$,

so the maximum sensitivity is reached in the wings of the $\sigma$ components. Taking the negative sign for $A_{1}^{V}$ in Eq. (16) one finally has

$R_{\mathrm{B}, 4}^{\prime}\left(\lambda_{\max }\right)=\mathrm{e}^{-1 / 2} C \frac{A_{0}^{V}}{A_{1}^{V}} g_{\mathrm{eff}} \lambda_{0}^{2}$.

Equation (17) tells us that the sensitivity of Stokes $V$ to $B$ is proportional to the shape ratio $A_{0}^{V} / A_{1}^{V}$, the Landé factor $g_{\text {eff }}$, and $\lambda_{0}^{2}$. Now the wavelength of the transition plays a crucial role, as it can effectively compensate for small shape ratios. Indeed, this explains why the infrared Fe I line at $1564.8 \mathrm{~nm}$ is more sensitive to $B$ than Fe I $630.25 \mathrm{~nm}$.

Figure 4 shows the maximum value of the integrated RF to magnetic field perturbations for the lines of Table 1 with $g_{\text {eff }} \neq 0$. Different symbols represent the quiet sun, penumbral, and umbral atmospheres. The following magnetic field strengths and inclinations have been assumed for the three models: $\left(2000 \mathrm{G}, 0^{\circ}\right),\left(1500 \mathrm{G}, 70^{\circ}\right)$, and $\left(2000 \mathrm{G}, 10^{\circ}\right)$, respectively. Very roughly, these values represent the conditions of plage regions and sunspot penumbrae and umbrae. We plot the maximum of the integrated RFs as a function of the parameter $\left(A_{0}^{V} / A_{1}^{V}\right) g_{\text {eff }} \lambda_{0}^{2}$. Also in this case, the phenomenological model explains the sensitivity of the lines to $B$ remarkably

\footnotetext{
5 Only the blue lobe of Stokes $V$, positive sign in Eq. (7), is considered here. Results for the red lobe are analogous.
}

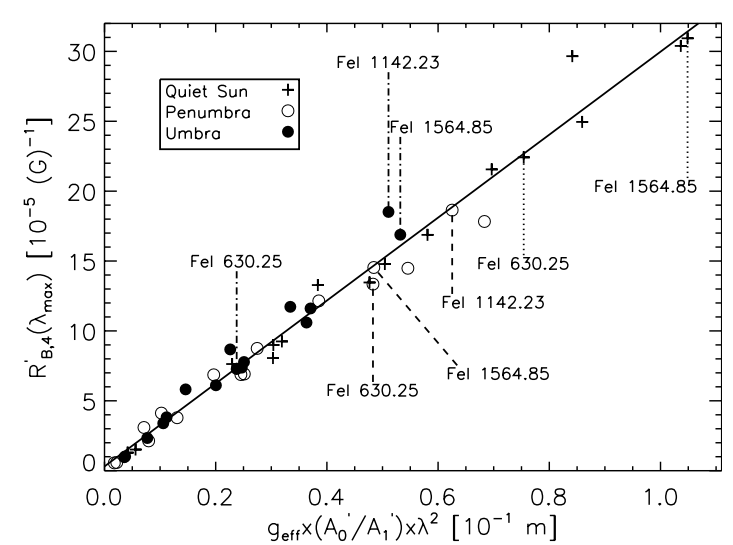

Fig. 4. Maximum values of the integrated RF to $B$ for the set of lines in Table 1 with $g_{\text {eff }} \neq 0$ vs $\left(A_{0}^{V} / A_{1}^{V}\right) g_{\text {eff }} \lambda_{0}^{2}$ (strong field regime). The sensitivities have been evaluated in the quiet sun (crosses), penumbral (circles), and umbral (filled circles) models. Dotted, dashed, and dash-dotted lines mark the most sensitive lines, Fe I $630.25 \mathrm{~nm}$, and Fe I $1564.85 \mathrm{~nm}$ in the quiet sun, penumbral, and umbral atmospheres, respectively.

well. We stress that any other combination of $B$ and $\gamma$ values in the atmospheric model would have led to the same linear relationship in Fig. 4 (with different values of the RFs and the shape ratio, of course). Thus, the figure can be used to gauge the sensitivity of any weak line to magnetic field perturbations in any atmosphere. To do that, it suffices to estimate the parameter $A_{0}^{V} / A_{1}^{V}$ of the Stokes $V$ profile of the line as it emerges from the atmosphere under consideration.

As can be seen in Fig. 4, the sensitivity to $B$ is larger in the quiet sun model. This can be traced to the higher temperatures (which lead to larger shape ratios) and the more vertical orientation of the magnetic field vector ${ }^{6}$ (which maximizes $A_{0}^{V}$ ). Lines in the umbra and the penumbra exhibit similar sensitivities. Although the penumbral model has higher temperatures (which would result in larger sensitivities), the more horizontal orientation of the field actually reduces the sensitivity.

\footnotetext{
${ }^{6}$ Although not explicitly written in Eq. (7), the Stokes $V$ amplitude depends on $\gamma$. This dependence shows up clearly when comparing the results for differently inclined magnetic fields, as in our example.
} 

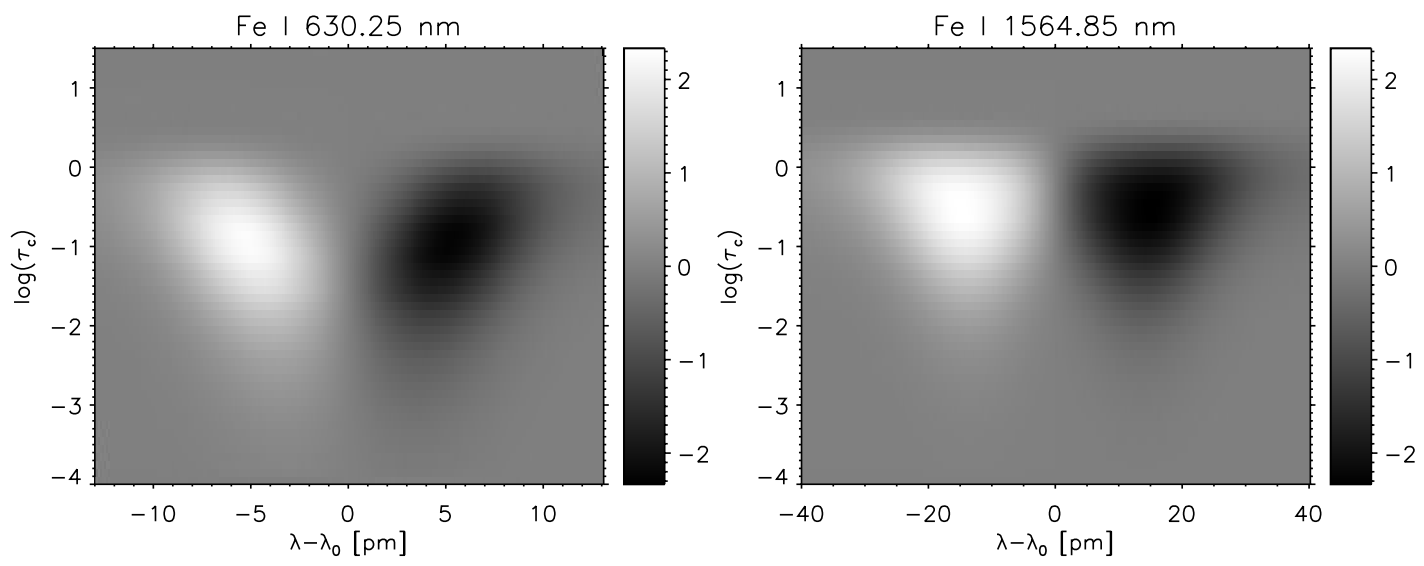

Fig. 5. Normalized RF of Stokes $V$ to $B$ multiplied by $\Delta \tau_{\mathrm{c}}\left[10^{-5} \mathrm{G}^{-1}\right]$ for Fe I $630.25 \mathrm{~nm}$ (left) and Fe I $1564.85 \mathrm{~nm}$ (right) in the HSRA model with a longitudinal magnetic field of $B=200 \mathrm{G}$. This corresponds to the weak field regime.

In Fig. 4, we have marked specific lines emerging from the penumbral and umbral models with dashed and dashdotted lines, respectively. The most sensitive line in the umbra and penumbra is Fe I $1142.32 \mathrm{~nm}$. Note also that the Fe I line at $1564.85 \mathrm{~nm}$ is observed to be more sensitive than Fe I $630.25 \mathrm{~nm}$ in all three models.

\subsection{Weak field regime}

Figure 5 shows the RFs of Stokes $V$ to $B$ for Fe I $630.25 \mathrm{~nm}$ and Fe I $1564.85 \mathrm{~nm}$ as emerging from the HSRA model with a constant longitudinal field of $200 \mathrm{G}$. Such a small field ensures that the lines are formed in the weak field regime. The atmospheric model adopted would be representative of quiet Sun internetwork fields (e.g., Khomenko et al. 2003). Contrary to the previous case, the RFs of Stokes $V$ to $B$ exhibit only two lobes in the weak field regime. The reason is that enhancements of the field strength increase the amplitude of the Stokes $V$ lobes, but do not shift them. Thus, RFs have positive blue lobes and negative red lobes.

The two lines probe similar layers as in the strong field regime, but now their sensitivities to $B$ are more or less the same. This finding, already reported by del Toro Iniesta \& Ruiz Cobo (1997), is somewhat surprising because the amplitude of Stokes $V$ in the weak field regime is proportional to $g_{\text {eff }} \lambda_{0}^{2}$. Thus, one would expect the infrared line to be much more sensitive to $B$ than the visible line.

Again we use the phenomenological model of Sect. 2.3 to explain these features. Differentiating Eq. (9) with respect to $B$, the RF of Stokes $V$ to constant perturbations of the field strength can be written as

$R_{B, 4}^{\prime}(\lambda)=-C g_{\text {eff }} \lambda_{0}^{2} \cos \gamma A_{0}^{*} \frac{\left(\lambda-\lambda_{0}\right)}{\left(A_{1}^{*}\right)^{2}} \exp \left[\frac{-\left(\lambda-\lambda_{0}\right)^{2}}{2\left(A_{1}^{*}\right)^{2}}\right]$,

and so the maximum value of the RF is

$R_{\mathrm{B}, 4}^{\prime}\left(\lambda_{\max }\right)=\mathrm{e}^{-1 / 2} C \cos \gamma \frac{A_{0}^{*}}{A_{1}^{*}} g_{\mathrm{eff}} \lambda_{0}^{2}$.

Formally, the response of Stokes $V$ to $B$ in the weak field regime is very similar to that in the strong field regime

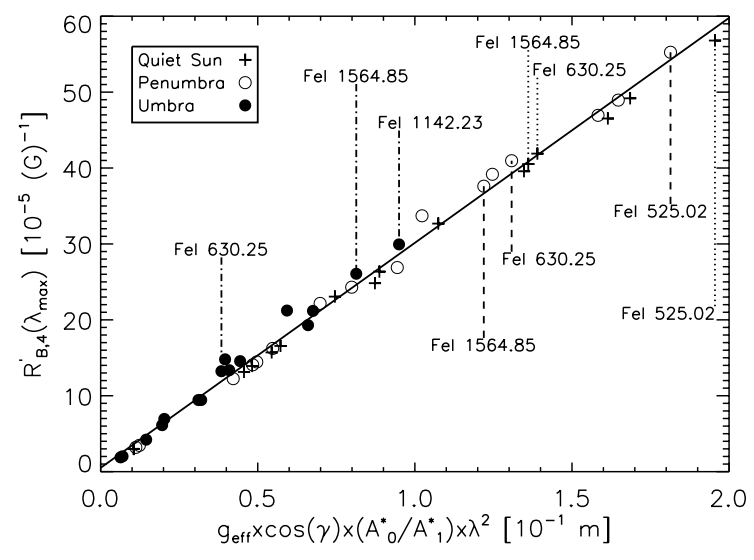

Fig. 6. Maximum values of the integrated RF to $B$ for the lines of Table 1 with $g_{\text {eff }} \neq 0$ vs the parameter $\left(A_{0}^{*} / A_{1}^{*}\right) \cos \gamma g_{\text {eff }} \lambda_{0}^{2}$ (weak field regime). The sensitivities have been evaluated in the quiet sun (crosses), penumbral (circles), and hot umbral (filled circles) models with a longitudinal magnetic field of $200 \mathrm{G}$. Dotted, dashed, and dashdotted lines mark specific transitions in the quiet sun, penumbral, and umbral models, respectively.

(compare Eqs. (19) with (17)), except for the explicit $\cos \gamma$ dependence. Now, however, the shape ratio $A_{0}^{*} / A_{1}^{*}$ refers to the Stokes $I$ profile, not to the Stokes $V$ profile. That is, the sensitivity to $B$ is determined by the sharpness of the intensity profile, together with the cosine of the field inclination $\gamma$, the Landé factor $g_{\text {eff }}$, and $\lambda_{0}^{2}$. Fe I $630.25 \mathrm{~nm}$ has a greater shape ratio than Fe I $1564.85 \mathrm{~nm}$. This compensates for the smaller $g_{\text {eff }} \lambda_{0}^{2}$ of the visible line, explaining why both lines exhibit more or less the same sensitivity in the quiet sun atmosphere.

In Fig. 6 we plot the maximum value of the integrated RF to field strength perturbations for the lines of Table 1 with $g_{\text {eff }} \neq 0$. For the three models considered we use a longitudinal magnetic field of $200 \mathrm{G}$. The most sensitive lines in the umbra and penumbra turn out to be Fe I $1142.32 \mathrm{~nm}$ and Fe I $525.02 \mathrm{~nm}$, respectively. The Fe I line at $1564.85 \mathrm{~nm}$ is more sensitive than Fe I $630.25 \mathrm{~nm}$ only in the umbral model. In hotter atmospheres, like the penumbral and quiet Sun models, a smaller shape ratio makes the infrared line slightly less sensitive to $B$ than the visible Fe I line at $630.25 \mathrm{~nm}$. 

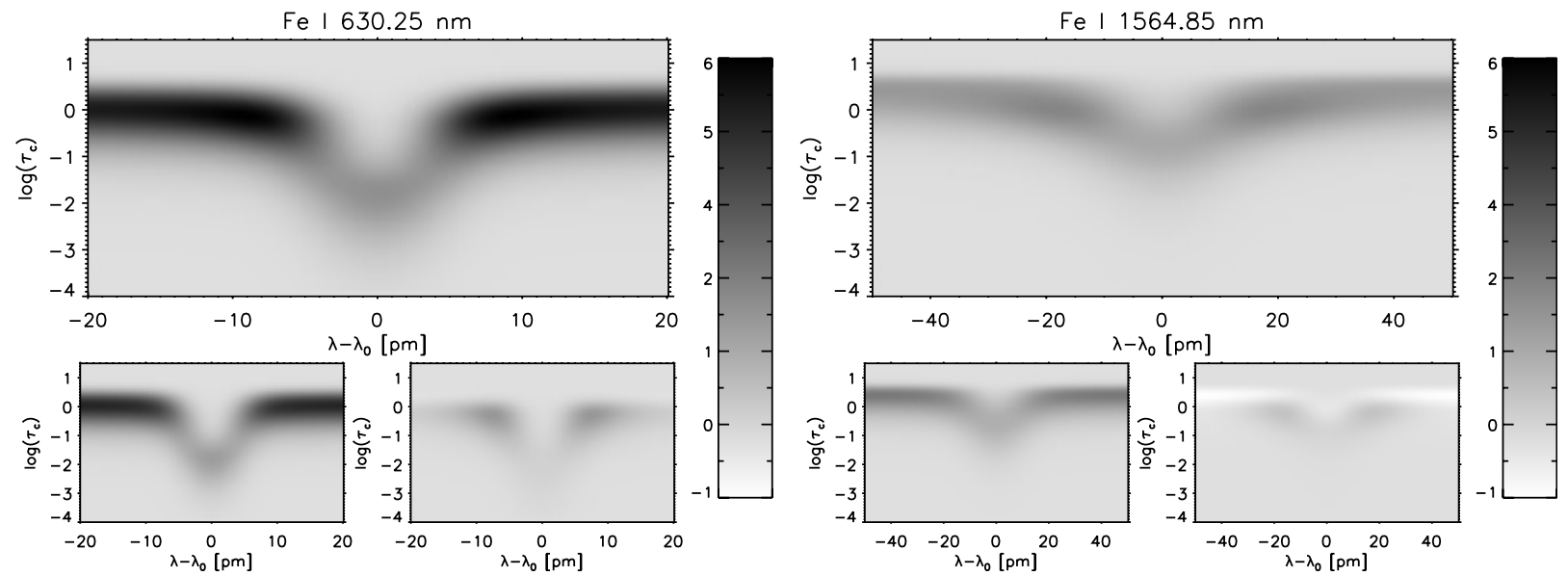

Fig. 7. Top: normalized RF of Stokes $I$ to $T$ multiplied by $\Delta \tau_{\mathrm{c}}\left[10^{-5} \mathrm{~K}^{-1}\right]$ for Fe I $630.25 \mathrm{~nm}$ (left) and Fe I $1564.85 \mathrm{~nm}$ (right) in the HSRA quiet sun model. Bottom: the two subpanels below each line display the first (left) and second (right) signed terms on the rhs of Eq. (2) contributing to the RF. That is, the first and third subpanels show how Stokes $I$ varies due to changes in the source function, while the second and fourth subpanels display how Stokes $I$ reacts to the opacity variations induced by temperature changes. Note that the gray scale is inverted in this figure, with black representing positive values and white negative values.

Comparing Figs. 4 and 6 it is clear that the sensitivities of Stokes $V$ to magnetic field perturbations in the weak field regime are larger than in the strong field regime. This result may look strange at first, but can be understood by noting that the shape ratio of Stokes $I\left(A_{0}^{*} / A_{1}^{*}\right)$ is about twice as large as that of Stokes $V\left(A_{0}^{V} / A_{1}^{V}\right)$ in the strong field case. According to Tables A.1 and A.3, $A_{1}^{*} \sim A_{1}^{V}$, while $A_{0}^{*} \sim 2 A_{0}^{V}$ for most of the lines. Physically, the larger sensitivity in the weak field regime results from the fact that changes in $B$ modify the amplitude of the Stokes $V$ profile, whereas in the strong field regime only the lobe separation varies. Due to the larger slope of Stokes $I$ in the line wing as compared with the slope of the $V$ profile (the parameters determining the sensitivity to $B$ in the two cases), changes in amplitude are much clearly seen than profile shifts. In practice, however, the inference of field strengths is less reliable in the weak field regime, because the amplitude of Stokes $V$ also depends on other parameters such as temperature and magnetic filling factor. Discriminating between these parameters and the magnetic field strength is often a difficult task.

\section{Sensitivity to temperature}

In this section we investigate how the intensity profiles and equivalent width of spectral lines react to temperature perturbations. The response of Stokes $V$ to $T$ is similar to that of Stokes $I$, because the circular polarization profile is the difference between the intensity profiles corresponding to righthanded and left-handed circularly polarized photons. As described by Eq. (2), changes in temperature induce modifications of both the propagation matrix and the source function. Below we demonstrate that the sensitivity of Stokes $I$ to temperature perturbations is mainly determined by variations of the source function $(\partial S / \partial T)$, whereas that of the equivalent width is mostly due to changes in absorption $(\partial \boldsymbol{K} / \partial T)$.

\subsection{Response of Stokes I to temperature}

The upper panels of Fig. 7 show the RFs of Stokes $I$ to temperature for the Fe I lines at $630.25 \mathrm{~nm}$ and $1564.85 \mathrm{~nm}$ as emerging from the HSRA quiet sun model. In the lower panels we plot the two (signed) terms on the rhs of Eq. (2) contributing to each RF: variations in the source function (left) and in the opacity (right). Inspection of these panels reveals that:

1. Variations in the source function result in a larger contribution to the RFs than opacity changes. Thus, the sensitivity of Stokes $I$ to temperature is dominated by changes in the source function, with opacity variations playing a less important role.

2. The variation of $S$ with $T$ is always positive: temperature enhancements increase the emissivity and therefore the emergent intensity, both in the continuum and in the line.

3. The term associated with variations of $\boldsymbol{K}$ with $T$ is predominantly positive except in the deepest photospheric layers. In the line, this reflects the well known fact that an increase of temperature leads to reduced absorption due to the larger ionization of iron. As a result of the smaller absorption, the emergent intensity increases. At continuum wavelengths, we find negative values deep in the atmosphere, indicating an increase of the continuum absorption after temperature enhancements. This phenomenon is better seen in the Fe I line at $1564.85 \mathrm{~nm}$ (fourth bottom panel).

Having discussed the various contributions to the sensitivity of Fe I $630.25 \mathrm{~nm}$ and Fe I $1564.85 \mathrm{~nm}$ to temperature, we summarize below the main features of the RFs displayed in the upper panels of Fig. 7:

1. The RFs are positive everywhere for the two lines. That is, enhancements of the temperature always increase the emergent intensity (in either the line, the continuum, or both), regardless of the atmospheric layer where the enhancement 
occurs. This is due to the fact that the main contribution to the RF is that associated with changes in the source function, which is always positive.

2. The visible line is sensitive to $T$ perturbations from $\log \tau_{\mathrm{c}} \sim$ 0.5 up to $\sim-3.0$ and the infrared line from $\log \tau_{\mathrm{c}} \sim 0.8$ to $\sim-1.5$. Therefore, Fe I $1564.85 \mathrm{~nm}$ is able to probe slightly deeper layers than Fe I $630.25 \mathrm{~nm}$, but it does not react to temperature changes in the mid-photosphere. The reason was explained in Sect. 2.2.

3. The two lines are sensitive to $T$ over a broader range of optical depths than to any other atmospheric parameter (compare Figs. 1, 3, and 5 with Fig. 7). The reason was explained by del Toro Iniesta \& Ruiz Cobo (1996): in LTE the source function depends only on temperature, and so the first term on the rhs of Eq. (2) is non-zero only for $x_{\mathrm{i}}=T$. As a result, the RFs to temperature have the slowest trend to zero with depth. This property makes it possible to obtain information on temperatures in layers where no information on other atmospheric parameters is reachable.

4. The infrared line is less sensitive to $T$ than the visible line.

To understand the difference in sensitivity between visible and infrared lines (item 4 of the list above) we apply again the phenomenological model of Sect. 2.3. Differenciating Eq. (6) with respect to $T$, the RF of Stokes $I$ to constant perturbations of $T$ can be written as

$$
\begin{aligned}
R_{T, 1}^{\prime}(\lambda)= & \frac{\partial A_{2}}{\partial T}-\left[\frac{\partial A_{0}}{\partial T}+\frac{\partial A_{1}}{\partial T} \frac{A_{0}}{A_{1}^{3}}\left(\lambda-\lambda_{0}\right)^{2}\right] \\
& \times \exp \left[\frac{-\left(\lambda-\lambda_{0}\right)^{2}}{2 A_{1}^{2}}\right],
\end{aligned}
$$

with extrema at

$$
\begin{aligned}
& \lambda_{\text {ext }}^{\mathrm{a}}=\lambda_{0} \pm A_{1} \sqrt{2-\frac{A_{1}}{A_{0}} \frac{\partial A_{0} / \partial T}{\partial A_{1} / \partial T},} \\
& \lambda_{\text {ext }}^{\mathrm{b}}=\lambda_{0}, \\
& \lambda_{\text {ext }}^{\mathrm{c}}=\lambda_{\mathrm{c}} .
\end{aligned}
$$

Here, $\lambda_{\mathrm{c}}$ stands for continuum wavelengths. At these locations, the RF has relative maxima or minima:

$$
\begin{aligned}
R_{T, 1}^{\prime}\left(\lambda_{\text {ext }}^{\mathrm{a}}\right)= & \frac{\partial A_{2}}{\partial T}-2 \frac{A_{0}}{A_{1}} \frac{\partial A_{1}}{\partial T} \\
& \times \exp \left[-1+\frac{1}{2} \frac{A_{1}}{A_{0}} \frac{\partial A_{0} / \partial T}{\partial A_{1} / \partial T}\right], \\
R_{T, 1}^{\prime}\left(\lambda_{\text {ext }}^{\mathrm{b}}\right)= & \frac{\partial A_{2}}{\partial T}-\frac{\partial A_{0}}{\partial T}, \\
R_{T, 1}^{\prime}\left(\lambda_{\text {ext }}^{\mathrm{c}}\right)= & \frac{\partial A_{2}}{\partial T} .
\end{aligned}
$$

Most neutral lines show the maximum value of the RF in the line wing $\left(\lambda_{\text {ext }}^{\mathrm{a}}\right)$. Very weak lines with optically thin cores have the maximum at the central wavelength $\left(\lambda_{\mathrm{ext}}^{\mathrm{b}}\right)$. Finally, singlyionized lines show a minimum at the central wavelength whilst $\lambda_{\text {ext }}^{\mathrm{a}}$ is complex (since the discriminant of Eq. (21) is negative). The latter have, of course, their maxima on the interval boundaries, that is, at continuum wavelengths $\left(\lambda_{\text {ext }}^{\mathrm{c}}\right)$. In summary, if we call $P_{\mathrm{T}}$ the maximum value of the RF for a given line, this

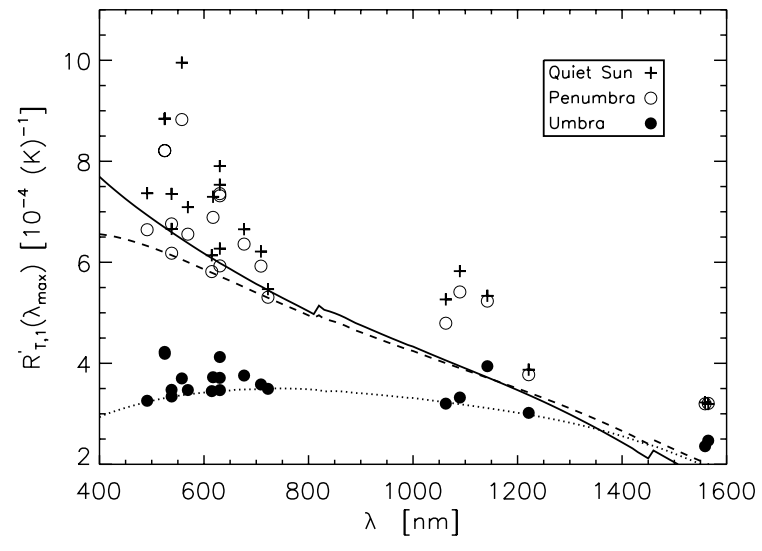

Fig. 8. Maximum value of the integrated RF of Stokes $I$ to $T$ for the lines of Table 1 vs. the wavelength of each transition. The sensitivities have been evaluated in the quiet Sun (crosses), penumbral (circles), and umbral (filled circles) models. Solid, dashed, and dotted curves represent the partial derivatives of the continuum intensity with respect to temperature $\left(\partial A_{2} / \partial T\right)$ in the quiet Sun, penumbral, and umbral models.

can be one of the three possibilities: $R_{T, 1}^{\prime}\left(\lambda_{\text {ext }}^{\mathrm{a}}\right), R_{T, 1}^{\prime}\left(\lambda_{\mathrm{ext}}^{\mathrm{b}}\right)$, or $R_{T, 1}^{\prime}\left(\lambda_{\text {ext }}^{\mathrm{c}}\right)$.

In all three cases the maximum value of the RF to $T$ is determined mostly by the variation of the continuum intensity with temperature, i.e., by $\partial A_{2} / \partial T$. This can be demonstrated as follows. Using the Eddington-Barbier approximation, we may write

$\frac{\partial A_{2}}{\partial T} \propto \frac{\partial B_{\lambda}\left[T\left(\tau_{\lambda}=1\right)\right]}{\partial T}$,

with $B_{\lambda}$ the Planck function evaluated at the temperature of the layer where $\tau_{\lambda}=1$. The three curves displayed in Fig. 8 represent the variation of $\partial A_{2} / \partial T$ with wavelength in the quiet sun, umbral and penumbral atmospheres. We also plot in this figure the maximum value of the integrated RF to $T$ for the lines of Table 1 in the same models. As can be seen, the term $\partial A_{2} / \partial T$ accounts for most of the sensitivity of the lines to temperature, independently of the atmospheric model or wavelength. Of course, a perfect match between the curves and the data points is not to be expected, because the curves display only one of the terms (the dominant one) contributing to the RF. It is apparent from the figure that the sensitivity of the lines tend to be smaller at longer wavelengths. This is because the derivative of the Planck function with $T$ decreases with $\lambda$ for typical solar temperatures, which explains why Fe I $630.25 \mathrm{~nm}$ is more sensitive to $T$ than the infrared Fe I line at $1564.85 \mathrm{~nm}$.

In Fig. 9 we consider all the terms contributing to the response of Stokes $I$ to $T$. Here we plot the maximum value of the integrated RF to temperature perturbations for the lines of Table 1 as a function of the parameter $P_{\mathrm{T}}$ (given by Eqs. (24), (25) or (26) as appropriate). Clearly, the phenomenological model does an excellent job in explaining the sensitivities of the various lines to temperature.

In general, the response of Stokes $I$ to $T$ is reduced in the umbral model. The low temperatures of this model cause smaller values of $\partial A_{2} / \partial T$ (see Fig. 8) and consequently smaller sensitivities. Consistent with our previous findings (cf. Fig. 7), 


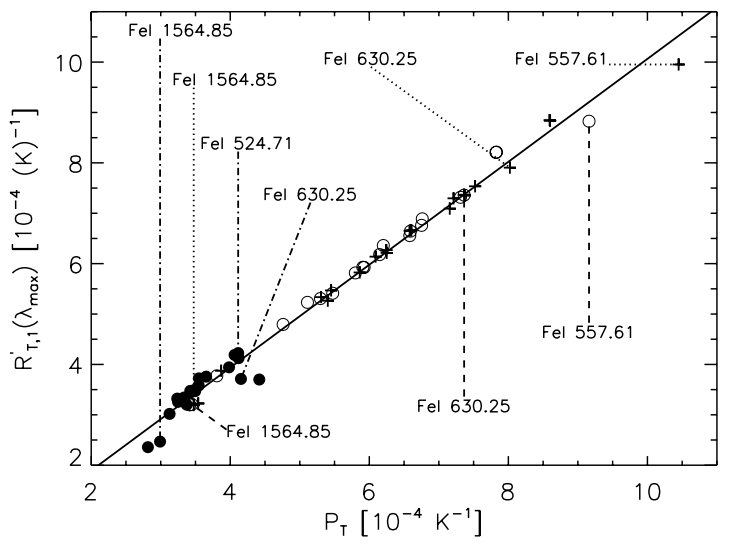

Fig. 9. Maximum value of the integrated RF of Stokes $I$ to $T$ for the lines of Table 1 vs. the parameter $P_{\mathrm{T}}$ (see text for details). Quiet Sun (crosses), penumbral (circles), and umbral (filled circles) atmospheres have been used. The most sensitive lines, Fe I $630.25 \mathrm{~nm}$, and Fe I $1564.85 \mathrm{~nm}$ in these models are marked with dotted, dashed, and dot-dashed lines, respectively.

Fe I $630.25 \mathrm{~nm}$ exhibits larger sensitivities than Fe I 1564.85 $\mathrm{nm}$ in all the models. Note, however, that the sensitivity of the infrared line is different from zero. Hence, there is a measurable change in the profile that can be used to retrieve the thermal structure of the photosphere. Figure 9 also shows that Fe I $557.61 \mathrm{~nm}$ is the most sensitive line of our set, disproving the general belief that Fe I $557.61 \mathrm{~nm}$ does not react to temperature changes.

\subsection{Response of equivalent width to temperature}

The equivalent width $W$ of a spectral line is defined as

$W=\Delta \sum_{i=1}^{q-1}\left[1-I\left(\lambda_{i}\right) / I_{c}\right]$

where $\Delta$ is the wavelength sampling interval, $I\left(\lambda_{i}\right)$ the intensity at each wavelength, and $I_{\mathrm{c}}$ the continuum intensity. Some authors (e.g., Moore et al. 1966; Gray 1992) use the change of $W$ with temperature as a diagnostic of the sensitivity of the lines to temperature perturbations.

Figure 10 shows the RFs of $W$ to $T$ as a function of $\log \tau_{\mathrm{c}}$ for Fe I 630.25 (solid line) and Fe I $1564.85 \mathrm{~nm}$ (dotted line) in the quiet Sun model. Both curves are double lobed. As explained by Ruiz Cobo \& del Toro Iniesta (1994), the shape of these RFs results from the competition between $\boldsymbol{K}$ and $\boldsymbol{S}$ in the final modification of $W$ (cf. Eq. (2)). A positive lobe coming from the derivative of $S$ with respect to $T$ dominates the behavior of the RFs in the deep layers, indicating that temperature enhancements in those layers increase the equivalent width due to the availability of more photons to be absorbed. The negative lobe in higher layers corresponds to the derivative of $\boldsymbol{K}$ with $T$, and implies a decrease of $W$ after an increase in temperature, i.e., a line weakening. Higher temperatures result in less Fe I atoms and, consequently, the equivalent width is reduced. Of course, since the same increase of temperature enhances the number of Fe II absorbers, lines like Fe II $614.93 \mathrm{~nm}$

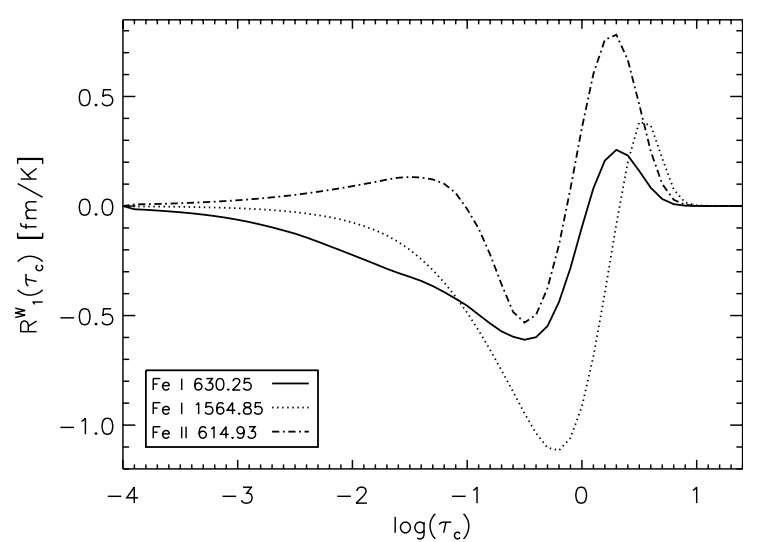

Fig. 10. Response function of the equivalent width to temperature perturbations for Fe I 630.25 (solid line), Fe I $1564.85 \mathrm{~nm}$ (dotted line), and Fe II $614.93 \mathrm{~nm}$ (dash-dotted line) in the quiet Sun model.

(dash-dotted line in Fig. 10) exhibit a positive lobe in mid and high photospheric layers.

A comparison of the RFs of $\mathrm{Fe} I 630.25 \mathrm{~nm}$ and Fe I $1564.85 \mathrm{~nm}$ depicted in Fig. 10 reveals two additional features: (a) the variation of $W$ with $T$ is larger for the infrared line than for the visible line ${ }^{7}$; and (b) the equivalent width of the infrared line is sensitive to temperature perturbations in deeper layers than the visible line.

According to our phenomenological model, the equivalent width of weak spectral lines can be written as

$W=\int\left(1-\frac{I}{A_{2}}\right) \mathrm{d} \lambda=\sqrt{2 \pi} \frac{A_{0} A_{1}}{A_{2}}$,

and so the derivative of $W$ with respect to $T$ is

$\frac{\partial W}{\partial T} \equiv P_{\mathrm{W}}=W\left(\frac{1}{A_{0}} \frac{\partial A_{0}}{\partial T}+\frac{1}{A_{1}} \frac{\partial A_{1}}{\partial T}-\frac{1}{A_{2}} \frac{\partial A_{2}}{\partial T}\right)$.

Therefore, as expected, variations of the equivalent width can be produced by changes in the line depth, the line width, and/or the continuum intensity. In Fig. 11 we plot the integrated RF of $W$ to $T$ for the lines of Table 1 in the quiet sun, umbral and penumbral atmospheres, against the parameter $P_{\mathrm{W}}$ defined above. Clearly, the phenomenological model succeeds in providing a good description of how the equivalent width reacts to temperature changes.

In the quiet sun and penumbral models, the variation of $W$ with $T$ is negative for all the $\mathrm{Fe} I$ lines and positive for the Fe II lines. This indicates that absorption processes dominate the behavior of $W$ : temperature enhancements decrease the number of neutrals and increase that of singly-ionized absorbers, i.e., the neutral lines are weakened and the singlyionized lines get strengthened. In the umbra, a few Fe I lines exhibit positive variations of $W$ with $T$; these lines have large excitation potentials, so it is difficult to populate their lower atomic levels in the cool umbral atmosphere. As a result, $\partial \boldsymbol{S} / \partial T$ dominates over $\partial \boldsymbol{K} / \partial T$, which explains why these lines become stronger after temperature enhancements.

\footnotetext{
${ }^{7} R_{T}^{\prime W}$, i.e., the integral of the RF of $W$ to $T$ with depth, is around -0.09 for Fe I $630.25 \mathrm{~nm}$ and -0.12 for Fe I $1564.85 \mathrm{~nm}$ in the quiet Sun model.
} 


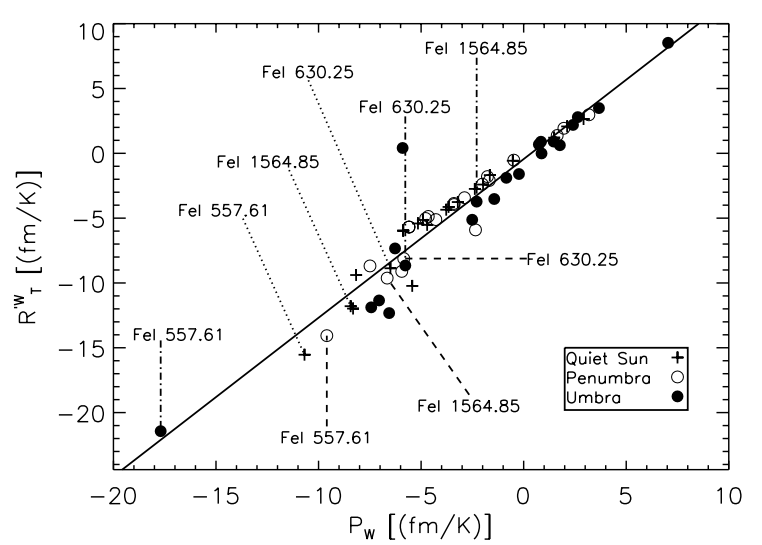

Fig. 11. Integrated RF of equivalent width to temperature perturbations vs. $P_{\mathrm{W}}$ for HSRA (crosses), penumbral (circles) and umbral (filled circles) model atmospheres.

Remarkably enough, Fe I $557.6 \mathrm{~nm}$ is the line showing the largest change of $W$ with $T$ in the three atmospheric models under consideration. This gives additional support to our claim that Fe I $557.6 \mathrm{~nm}$ can be used to infer the thermal stratification of the solar atmosphere. Figure 11 also demonstrates that the infrared Fe I line at $1564.85 \mathrm{~nm}$ exhibits larger changes of $W$ than Fe I $630.25 \mathrm{~nm}$ in all models except in the umbra, where the lower atomic level of the infrared line is not well populated.

\section{Discussion}

There are many ways to derive the physical properties of stellar atmospheres from spectral line profiles, but the question still remains which lines are best suited to retrieve a given atmospheric parameter. We have addressed this question by analyzing the response functions of a number of weak lines commonly used in solar physics. Our analysis provides a better understanding of the diagnostic capabilities of these lines.

Describing the shapes of Stokes $I$ and $V$ in terms of simple parameters (such as line widths and depths), we have been able to explain why different lines exhibit different sensitivities to the same atmospheric parameter (e.g., LOS velocities, magnetic field strengths or temperatures). By sensitivity we mean the change of the intensity and circular polarization signal at a given wavelength caused by a height-independent perturbation of an atmospheric parameter. If the sensitivity is small, the changes in the emergent spectrum may not be detectable depending on the noise of the observations. Thus, it is always advisable to select lines having large sensitivities to a given parameter, in order to ensure that the subtle variations it produces in the spectrum are not buried by the noise.

\subsection{Visible vs. infrared lines}

The formation height of spectral lines depends primarily on the excitation potential and oscillator strength of the atomic transition, together with the temperature of the atmosphere, rather than on the central wavelength of the transition. Therefore, it is not possible to draw general conclusions about differences between visible and infrared lines; each line has to be investigated separately. However, we have identified some trends that explain the peculiarities of many lines in the two spectral regions:

1. The thermal width of the lines (represented roughly by $A_{1}$ ) increases linearly with wavelength. Therefore, the intensity and circular polarization profiles of infrared lines are broader, leading to a general reduction of the sensitivity to LOS velocities and magnetic fields (in the weak field limit).

2. The ratio of Zeeman splitting to thermal width increases linearly with wavelength. Thus, changes in the magnetic field strength are much easier to detect in the infrared when the field is sufficiently large.

3. In general, the excitation potential of infrared lines is rather large, implying that high temperatures are necessary to populate their lower atomic levels. As a consequence, many infrared lines are not formed in the mid and upper photospheric layers, where the temperatures are low. Fe I $1142.23 \mathrm{~nm}$ is an exception to this rule. With an excitation potential of $2.2 \mathrm{eV}$, this line is able to sample higher atmospheric layers than Fe I $630.25 \mathrm{~nm}$.

4. Intensity contrasts induced by temperature fluctuations are smaller in the infrared because the Planck function changes less with $T$ at longer wavelengths. This is the reason for the lower sensitivity of infrared lines to temperature as compared with visible lines. However, the variation of $W$ with $T$ is generally larger for infrared lines, due to their broader intensity profiles.

Since the continuum opacity reaches a minimum in the solar photosphere at about $1.6 \mu \mathrm{m}$, one could expect to obtain extra information on deep layers by studying infrared lines close to this wavelength. The detailed comparison between Fe I $630.25 \mathrm{~nm}$ and Fe I $1564.85 \mathrm{~nm}$ presented here shows that such an extra information is not that significant except perhaps for temperature: the deepest layer to which the infrared line is sensitive is at most $70 \mathrm{~km}$ deeper than the corresponding one for the visible line. The main advantage of the infrared, then, is not in going deeper but in having a combination of large sensitivity to magnetic fields and reasonable sensitivity to velocities in a narrow range of layers. This allows for a good discrimination of the different structures that may coexist in the resolution element as, for example, in the case of sunspot penumbrae.

Both visible and infrared lines have their strengths and limitations. Simultaneous observations of the Sun in the visible and infrared would certainly improve the diagnostic capabilities of a single spectral range alone. This kind of observations are now possible with the new generation of solar polarimeters, such as TIP and POLIS, operated simultaneously at the Vacuum Tower Telescope of Teide Observatory, and the SpectroPolarimeter for Infrared and Optical Ranges (SPINOR), to be installed at the Dunn Solar Telescope of NSO/Sacramento Peak Observatory (Socas-Navarro et al. 2005).

\subsection{Temperature insensitive lines}

One of the main results of this work is that lines considered to be temperature insensitive do indeed show a significant temperature dependence. Fe I $557.6 \mathrm{~nm}$ belongs to this group of 
lines. In Rowland's tables (cf. Moore et al. 1966), the equivalent width of Fe I $557.6 \mathrm{~nm}$ is mentioned not to change much from the quiet sun to sunspot umbrae (two atmospheres with rather different temperatures). This is probably the reason why Fe I $557.6 \mathrm{~nm}$ is regarded as the prototype of temperature insensitive lines by most solar physicists. Recent observations, however, demonstrate that the equivalent width of this line is at least 30\% larger in the umbra than in the quiet sun (see Fig. 5 of Tritschler et al. 2004). The high spatial and spectral resolution observations of Tritschler et al. are in agreement with the results of this work, where we see that Fe I $557.6 \mathrm{~nm}$ undergoes the largest change of equivalent width with $T$ among the various lines in the sample. Of course, there are other lines not considered here that show larger temperature sensitivities. Molecular lines are a good example: many of them show up only in the cool umbrae, disappearing completely in hotter atmospheres due to the dissociation of the parent molecules.

Another line which is often quoted to be a poor diagnostics of temperature is Fe I $1564.85 \mathrm{~nm}$. Our analysis shows that, due to the smaller change of the Planck function with $T$ at longer wavelengths, the intensity profiles of infrared lines are less influenced by temperature variations than visible lines. In this sense, Fe I $1564.85 \mathrm{~nm}$ is indeed not as appropriate as, e.g., Fe I $630.25 \mathrm{~nm}$ for retrieving the thermal stratification of the solar photosphere. However, Fe I $1564.85 \mathrm{~nm}$ does react to temperature perturbations, as demonstrated by Figs. 9 and 11. In particular, the change of the equivalent width of $\mathrm{Fe} I$ $1564.85 \mathrm{~nm}$ with $T$ is larger than that of Fe I $630.25 \mathrm{~nm}$ in the quiet sun and penumbral models.

\subsection{Applicability}

The calculations have been carried out in three model atmospheres representing the conditions of sunspot umbrae and penumbrae, as well as those of the quiet sun. For simplicity, we have assumed constant (height-independent) magnetic fields and LOS velocities in these models. Such an assumption has allowed us to characterize the sensitivity of spectral lines to a given atmospheric parameter by means of a single number (the maximum value of the integrated response function across the line profile). That is, our approach neglects possible variations of the atmospheric parameters with height. Since we know that vertical gradients exist in the solar atmosphere (e.g., in sunspot penumbrae), it is important to discuss whether the results of this paper would be applicable to atmospheres featuring gradients of the physical quantities.

The strength of our phenomenological model lies in that it identifies the physical mechanism(s) responsible for the sensitivity of spectral lines. The response to LOS velocities, for instance, is determined by the sharpness of the intensity profile, and this is true in any atmospheric model. Gradients of LOS velocity do modify the shape of Stokes $I$ but, unless they are very strong, the intensity profile can always be described reasonably well by a gaussian (with different parameters, of course). Thus, a preliminary selection of lines can be done by means of the recipes obtained in this work. In the real sun, the sensitivities of the lines will be different from those predicted by our model, but they will differ by the same factor for all the lines. Hence, Fe I $524.71 \mathrm{~nm}$ will always be the most sensitive line of our set to LOS velocities, even if the atmosphere features variations of this quantity with height. The same applies to the response of the lines to other atmospheric parameters.

At this point it is important to recall that the sensitivity of spectral lines to LOS velocities and magnetic fields is determined by the shape ratios of Stokes $I$ and Stokes $V$. The shape ratio measures the slope of the corresponding Stokes profile. This quantity can be significantly modified by the instrument used to take the observations. In fact, spectrographs or spectropolarimeters with poor spectral resolutions may degrade the diagnostic potential of the lines by broadening their intensity and circular polarization profiles. In other words: the same lines may show different sensitivities when observed with different instruments or telescopes. This fact is often disregarded.

\subsection{The most sensitive lines of the set}

The sensitivity of spectral lines depends on the values of the physical parameters of the atmosphere where they are formed. Hence, lines which are universally the most sensitive to a given parameter regardless of the model atmosphere do not exist. Anyway, one can identify for each atmospheric quantity some spectral lines in Table 1 which have a high response:

- If one is interested in retrieving temperatures from line profiles, Fe I $557.61 \mathrm{~nm}, \mathrm{Fe}$ I $525.02 \mathrm{~nm}$, and Fe I $524.71 \mathrm{~nm}$ display quite large changes after subtle modifications of the temperature.

- As explained in Sect. 6.1, infrared lines usually exhibit lower sensitivity to LOS velocity as compared with the visible lines. Therefore, the lines with better response to $v_{\mathrm{LOS}}$ are usually in the visible part of the solar spectrum. In fact, the most sensitive lines of our set are Fe I $524.71 \mathrm{~nm}$, Fe I $525.02 \mathrm{~nm}$, and Ni I $676.70 \mathrm{~nm}$. On the other hand, LOS velocities are often estimated from measurements of line bisectors. Magnetically insensitive lines $\left(g_{\text {eff }}=0\right)$ with a low response to temperature perturbations are then advisable. In this case, one should prefer Ni I $491.21 \mathrm{~nm}$, Fe I $709.04 \mathrm{~nm}$, or Fe I $569.15 \mathrm{~nm}$.

- Stokes V from Fe I $1142.23 \mathrm{~nm}$, Fe I $525.02 \mathrm{~nm}$, and Fe I $1564.85 \mathrm{~nm}$ show the largest reaction to field strength modifications in the strong field regime. In the weak field regime, Fe I $1142.23 \mathrm{~nm}$ and Fe I $525.02 \mathrm{~nm}$ are also the most sensitive lines of the set.

\section{Summary}

We have characterized the response of weak spectral lines to LOS velocities, magnetic fields, and temperatures by means of their response functions evaluated in three model that represent the conditions of the quiet sun, sunspot umbrae, and penumbrae. In addition, we have developed a simple phenomenological model that is able to predict the response of any weak line to these atmospheric parameters. Thus, for the first time, we have at our disposal simple rules to select the most appropriate line for the problem we are interested in. 
Not surprisingly, the response of Stokes I to LOS velocities increases with both the sharpness of the profile and the wavelength. In general, visible lines show higher sensitivities than infrared lines because their intensity profiles are sharper.

The response of Stokes $V$ to magnetic field strength is formally the same in the strong and weak field limits, because it depends on the sharpness of the Stokes $V$ or Stokes $I$ profiles, as well as on the amount of Zeeman splitting. The strong variation of the Zeeman splitting with wavelength usually compensates for the broader profiles of infrared lines, making them the lines of choice for determining magnetic field strengths.

We have shown that the main contribution to the sensitivity of Stokes $I$ to temperature is the variation in the source function with $T$, and that opacity changes play a less important role. Since variation of the Planck function with $T$ is smaller at longer wavelengths, infrared lines are less affected by temperature perturbations than visible lines. However, they show a large modification of the equivalent width with $T$.

Our set of lines includes some lines that are often quoted to be temperature insensitive, such as Fe I $557.61 \mathrm{~nm}$. We have demonstrated that this line in particular exhibits the highest temperature sensitivity among the various lines considered in this work. In fact, one cannot speak of temperature insensitive lines: even if the absorption does not change much after temperature variations, the source function will always change, leading to detectable effects in the emergent spectrum.

Ideally, one would like to use a spectral line that shows very high sensitivity to all atmospheric parameters at the same time, but this line does not exist. Visible and infrared lines have both advantages and limitations. It is desirable, then, to combine different lines in order to characterize the physical properties of the solar atmosphere more reliably. Simultaneous observations of visible and infrared lines are now possible in several telescopes, and this will undoubtedly open a new era of solar physics research.

Acknowledgements. Discussions with Basilio Ruiz Cobo and Helmold Schleicher are gratefully acknowledged. This work was supported by the Programa Ramón y Cajal of the Spanish Ministerio de Educación y Ciencia, project AyA2001-1649 of the Spanish Programa Nacional de Astronomía y Astrofísica, and projects ESP2002-04256C04-01 and ESP2003-07735-C04-03 of the Programa Nacional del Espacio, partly using European FEDER funds.

\section{References}

Battrick, B., \& Sawaya-Lacoste, H. 2001, Solar Encounter: The First Solar Orbiter Workshop, ESA SP-493

Collados, M., Martínez Pillet, V., Ruiz Cobo, B., \& del Toro Iniesta, J. C. 1994, A\&A, 291, 622

Collados, M., Rodríguez Hidalgo, I., Bellot Rubio, L. R., Ruiz Cobo, B., \& Soltau, D. 1999, Mitt. Astron. Gesell., 15, 20

Elmore, D. F., Lites, B. W., Tomczyk, S., et al. 1992, Proc. SPIE, 1746, 22
Frutiger, C. 2000, Ph.D. Thesis, ETH Zürich

Gandorfer, A. M., Solanki, S. K., Schüssler, M., et al. 2004, SPIE, 5489,732

Gingerich, O., Noyes, R. W., Kalkofen, W., \& Cuny, Y. 1971, Sol. Phys., 18, 347

Gray, D. 1992, The observation and analysis of stellar photospheres, 2nd Ed. (Cambrigde: Cambridge University Press)

Khomenko, E. V., Collados, M., Solanki, S. K., Lagg, A., \& Trujillo Bueno, J. 2003, A\&A, 408, 1115

Landi degl'Innocenti, E. 1992, in Solar Observations: Techniques and Interpretation, ed. F. Sánchez, M. Collados, \& M. Vázquez (Cambridge: Cambridge Univ. Press), 1, 73

Landi degl'Innocenti, E., \& Landi degl'Innocenti, M. 1977, A\&A, 56, 111

Lites, B. W., Elmore, D. F., \& Streander, K. V. 2001, ASP Conf. Ser., 236, 33

Marsch, E., Antonucci, E., Bochsler, P., et al. 2002, Adv. Space Res., 29, 2027

Martínez Pillet, V., Collados, M., Sánchez Almeida, et al. 1999, ASP Conf. Ser., 183, 264

Mein, P. 1971, Sol. Phys., 20, 3

Moore, C. E., Minnaert, M. G. J., \& Houtgast, J. 1966, The Solar Spectrum from $2935 \AA$ to $8770 \AA$ A, National Bureau of Standards Monograph (Washington: US Government Printing Office)

Rüedi, I., Solanki, S. K., Livingston, W., \& Harvey, J. 1995, A\&A, 113, 91

Rüedi, I., Solanki, S. K., Keller, C. U., \& Frutiger, C. 1998, A\&A, 338, 1089

Ruiz Cobo, B., \& del Toro Iniesta, J. C. 1992, ApJ, 398, 375

Ruiz Cobo, B., \& del Toro Iniesta, J. C. 1994, A\&A, 283, 129

Scherrer, P. H. 2002, BAAS, 34, 735

Scherrer, P. H., Bogart, R. S., Bush, R. I., et al. 1995, Sol. Phys., 162, 129

Schmidt, W., Beck, C., Kentischer, T., Elmore, D. F., \& Lites, B. W. 2003, AN, 324, 300

Socas-Navarro, H., Elmore, D. F., \& Lites, B. W. 2005, Sol. Phys., submitted

Solanki, S. K., Pantellini, F. G. E., \& Stenflo, J. O. 1987, Sol. Phys., 107, 57

Solanki, S. K., Biémont, E., \& Mürset, U. 1990, A\&AS, 83, 307

Solanki, S. K., Rüedi, I., \& Livingston, W. 1992, A\&A, 263, 312

Solanki, S. K., Gandorfer, A. M., Schüssler, M., et al. 2003, Proc. SPIE, 4853, 129

del Toro Iniesta, J. C. 2003, Introduction to Spectropolarimetry (Cambridge: Cambridge Univ. Press)

del Toro Iniesta, J. C., Tarbell, T. D., \& Ruiz Cobo, B. 1994, ApJ, 436, 400

del Toro Iniesta, J. C., \& Ruiz Cobo, B. 1996, Sol. Phys., 164, 169

del Toro Iniesta, J. C., \& Ruiz Cobo, B. 1997, in Forum THEMIS: Science with THEMIS, ed. N. Mein, \& S. Sahal-Bréchot (Paris: Obs. de Paris), 93

Tritschler, A., Schlichenmaier, R., Bellot Rubio, L. R., and the KAOS team 2004, A\&A, 415, 717

Vela Villahoz, E., Sánchez Almeida, J., \& Wittmann, A. D. 1994, A\&A, 103, 293

von Klüber, H. 1948, ZAp, 24, 121 


\section{Online Material}


D. Cabrera Solana et al.: Sensitivity of weak spectral lines, Online Material p 2

\section{Appendix A: Tables}


D. Cabrera Solana et al.: Sensitivity of weak spectral lines, Online Material $p 3$

Table A.1. Weak line model parameters and their derivatives for the quiet Sun model. $A_{0}$ and $A_{1}$ are the residual intensity and the width of Stokes $I$ in the absence of magnetic fields, while $A_{2}$ is the continuum intensity. $A_{0}^{V}$ and $A_{1}^{V}$ are the amplitude and width of the Stokes $V$ lobes in the strong field regime. $A_{0}^{*}$ and $A_{1}^{*}$ are the depth and the width of Stokes $I$ in the weak field approximation $(B=200 \mathrm{G})$.

\begin{tabular}{|c|c|c|c|c|c|c|c|c|c|c|c|}
\hline Species & $\lambda_{0}[\mathrm{~nm}]$ & $A_{0}$ & $A_{1}[\mathrm{pm}]$ & $A_{2}$ & $\partial A_{0} / \partial T\left[\mathrm{~K}^{-1}\right]$ & $\partial A_{1} / \partial T[\mathrm{pm} / \mathrm{K}]$ & $\partial A_{2} / \partial T\left[\mathrm{~K}^{-1}\right]$ & $A_{0}^{V}$ & $A_{1}^{V}[\mathrm{pm}]$ & $A_{0}^{*}$ & $A_{1}^{*}[\mathrm{pm}]$ \\
\hline Ni I & 491.20 & 0.54 & 2.72 & 1.00 & $+1.26 \times 10^{-4}$ & $-5.27 \times 10^{-4}$ & $6.95 \times 10^{-4}$ & 0 & 0 & 0 & 0 \\
\hline $\mathrm{Fe} \mathrm{I}$ & 524.71 & 0.79 & 3.13 & 1.00 & $+6.73 \times 10^{-5}$ & $-1.16 \times 10^{-3}$ & $6.70 \times 10^{-4}$ & 0.39 & 3.12 & 0.76 & 3.17 \\
\hline $\mathrm{Fe} \mathrm{I}$ & 525.02 & 0.78 & 3.11 & 1.00 & $+5.12 \times 10^{-5}$ & $-1.13 \times 10^{-3}$ & $6.70 \times 10^{-4}$ & 0.39 & 3.10 & 0.76 & 3.20 \\
\hline $\mathrm{Fe}_{\mathrm{I}}$ & 537.96 & 0.63 & 3.17 & 1.00 & $+1.04 \times 10^{-4}$ & $-7.39 \times 10^{-4}$ & $6.61 \times 10^{-4}$ & 0.26 & 2.47 & 0.63 & 3.18 \\
\hline Ti II & 538.10 & 0.57 & 3.21 & 1.00 & $+5.36 \times 10^{-4}$ & $+1.24 \times 10^{-4}$ & $6.61 \times 10^{-4}$ & 0.22 & 2.45 & 0.56 & 3.23 \\
\hline $\mathrm{Fe} \mathrm{I}$ & 557.61 & 0.80 & 5.32 & 1.00 & $+4.52 \times 10^{-4}$ & $-4.78 \times 10^{-3}$ & $6.62 \times 10^{-4}$ & 0 & 0 & 0 & 0 \\
\hline $\mathrm{Fe} I$ & 569.15 & 0.47 & 3.24 & 1.00 & $+2.23 \times 10^{-5}$ & $-7.80 \times 10^{-4}$ & $6.40 \times 10^{-4}$ & 0 & 0 & 0 & 0 \\
\hline Fe II & 614.93 & 0.41 & 3.70 & 1.00 & $+5.70 \times 10^{-4}$ & $-7.80 \times 10^{-5}$ & $6.09 \times 10^{-4}$ & 0.19 & 3.23 & 0.40 & 3.74 \\
\hline $\mathrm{Fe} \mathrm{I}$ & 617.33 & 0.65 & 3.61 & 1.00 & $+9.69 \times 10^{-6}$ & $-8.85 \times 10^{-4}$ & $6.08 \times 10^{-4}$ & 0.32 & 3.59 & 0.63 & 3.71 \\
\hline $\mathrm{Fe} \mathrm{I}$ & 630.25 & 0.68 & 4.61 & 1.00 & $+2.10 \times 10^{-4}$ & $-2.44 \times 10^{-3}$ & $6.06 \times 10^{-4}$ & 0.33 & 4.40 & 0.66 & 4.72 \\
\hline $\mathrm{Fe}_{\mathrm{I}}$ & 630.35 & 0.06 & 3.17 & 1.00 & $-2.57 \times 10^{-5}$ & $-3.59 \times 10^{-4}$ & $5.99 \times 10^{-4}$ & 0.03 & 2.97 & 0.06 & 3.22 \\
\hline Ti I & 630.38 & 0.09 & 3.13 & 1.00 & $-1.54 \times 10^{-4}$ & $-5.92 \times 10^{-5}$ & $5.99 \times 10^{-4}$ & 0.03 & 3.03 & 0.09 & 3.16 \\
\hline Ni I & 676.78 & 0.72 & 4.49 & 1.00 & $+1.79 \times 10^{-4}$ & $-1.16 \times 10^{-3}$ & $5.74 \times 10^{-4}$ & 0.35 & 4.15 & 0.7 & 4.53 \\
\hline $\mathrm{Fe} \mathrm{I}$ & 709.04 & 0.50 & 4.17 & 1.00 & $+2.59 \times 10^{-5}$ & $-9.06 \times 10^{-4}$ & $5.54 \times 10^{-4}$ & 0 & 0 & 0 & 0 \\
\hline Fe II & 722.45 & 0.15 & 3.83 & 1.00 & $+3.15 \times 10^{-4}$ & $-2.99 \times 10^{-4}$ & $5.45 \times 10^{-4}$ & 0 & 0 & 0 & 0 \\
\hline Si I & 1062.76 & 0.44 & 11.24 & 1.00 & $+2.54 \times 10^{-4}$ & $-6.55 \times 10^{-3}$ & $4.24 \times 10^{-4}$ & 0.21 & 10.89 & 0.43 & 11.45 \\
\hline $\mathrm{Fe} \mathrm{I}$ & 1089.63 & 0.31 & 5.74 & 1.00 & $-1.91 \times 10^{-4}$ & $-8.52 \times 10^{-4}$ & $3.96 \times 10^{-4}$ & 0.15 & 5.72 & 0.29 & 5.99 \\
\hline $\mathrm{Fe} \mathrm{I}$ & 1142.23 & 0.54 & 7.42 & 1.00 & $-5.95 \times 10^{-5}$ & $-2.44 \times 10^{-3}$ & $3.77 \times 10^{-4}$ & 0.27 & 8.42 & 0.51 & 7.81 \\
\hline $\mathrm{Fe}_{\mathrm{I}}$ & 1221.33 & 0.12 & 6.77 & 1.00 & $-4.59 \times 10^{-5}$ & $-1.84 \times 10^{-3}$ & $3.39 \times 10^{-4}$ & 0.06 & 6.74 & 0.10 & 7.84 \\
\hline $\mathrm{Fe} I$ & 1558.83 & 0.31 & 11.66 & 1.00 & $+3.87 \times 10^{-6}$ & $-8.36 \times 10^{-3}$ & $1.89 \times 10^{-4}$ & 0.16 & 11.33 & 0.30 & 12.32 \\
\hline $\mathrm{Fe} \mathrm{I}_{\mathrm{I}}$ & 1564.85 & 0.31 & 10.95 & 1.00 & $-5.26 \times 10^{-5}$ & $-6.85 \times 10^{-3}$ & $1.84 \times 10^{-4}$ & 0.16 & 10.83 & 0.26 & 13.80 \\
\hline
\end{tabular}

Table A.2. Same as Table A.1, for the penumbral model.

\begin{tabular}{|c|c|c|c|c|c|c|c|c|c|c|c|}
\hline Species & $\lambda_{0}[\mathrm{~nm}]$ & $A_{0}$ & $A_{1}[\mathrm{pm}]$ & $A_{2}$ & $\partial A_{0} / \partial T\left[\mathrm{~K}^{-1}\right]$ & $\partial A_{1} / \partial T[\mathrm{pm} / \mathrm{K}]$ & $\partial A_{2} / \partial T\left[\mathrm{~K}^{-1}\right]$ & $A_{0}^{V}$ & $A_{1}^{V}[\mathrm{pm}]$ & $A_{0}^{*}$ & $A_{1}^{*}[\mathrm{pm}]$ \\
\hline Ni I & 491.20 & 0.46 & 2.49 & 0.83 & $+1.74 \times 10^{-4}$ & $-4.88 \times 10^{-4}$ & $6.34 \times 10^{-4}$ & 0 & 0 & 0 & 0 \\
\hline $\mathrm{Fe} I$ & 524.71 & 0.70 & 3.00 & 0.85 & $+1.74 \times 10^{-4}$ & $-1.27 \times 10^{-3}$ & $6.20 \times 10^{-4}$ & 0.14 & 2.00 & 0.69 & 3.04 \\
\hline $\mathrm{Fe} \mathrm{I}$ & 525.02 & 0.69 & 2.98 & 0.85 & $+1.62 \times 10^{-4}$ & $-1.25 \times 10^{-3}$ & $6.20 \times 10^{-4}$ & 0.18 & 2.14 & 0.67 & 3.06 \\
\hline $\mathrm{Fe} \mathrm{I}$ & 537.96 & 0.56 & 2.95 & 0.86 & $+1.50 \times 10^{-4}$ & $-7.25 \times 10^{-4}$ & $6.16 \times 10^{-4}$ & 0.07 & 2.00 & 0.56 & 2.96 \\
\hline Ti II & 538.10 & 0.48 & 2.94 & 0.86 & $+4.99 \times 10^{-4}$ & $+2.18 \times 10^{-4}$ & $6.15 \times 10^{-4}$ & 0.06 & 2,04 & 0.48 & 2.95 \\
\hline $\mathrm{Fe} \mathrm{I}$ & 557.61 & 0.68 & 5.21 & 0.86 & $+4.51 \times 10^{-4}$ & $-4.42 \times 10^{-3}$ & $6.25 \times 10^{-4}$ & 0 & 0 & 0 & 0 \\
\hline $\mathrm{Fe} \mathrm{I}$ & 569.15 & 0.43 & 2.98 & 0.87 & $+6.58 \times 10^{-5}$ & $-7.25 \times 10^{-4}$ & $6.02 \times 10^{-4}$ & 0 & 0 & 0 & 0 \\
\hline $\mathrm{Fe}$ II & 614.93 & 0.34 & 3.34 & 0.88 & $+5.49 \times 10^{-4}$ & $+7.38 \times 10^{-5}$ & $5.80 \times 10^{-4}$ & 0.04 & 2.81 & 0.33 & 3.38 \\
\hline $\mathrm{Fe} \mathrm{I}$ & 617.33 & 0.60 & 3.40 & 0.88 & $+7.87 \times 10^{-5}$ & $-9.41 \times 10^{-4}$ & $5.80 \times 10^{-4}$ & 0.15 & 2.56 & 0.58 & 3.50 \\
\hline $\mathrm{Fe} \mathrm{I}$ & 630.25 & 0.61 & 4.41 & 0.88 & $+2.44 \times 10^{-4}$ & $-2.24 \times 10^{-3}$ & $5.82 \times 10^{-4}$ & 0.14 & 2.89 & 0.60 & 4.53 \\
\hline $\mathrm{Fe} \mathrm{I}$ & 630.35 & 0.06 & 2.82 & 0.89 & $-1.85 \times 10^{-5}$ & $-3.09 \times 10^{-4}$ & $5.72 \times 10^{-4}$ & 0.01 & 2.53 & 0.05 & 2.88 \\
\hline Ti I & 630.38 & 0.10 & 2.80 & 0.89 & $-1.59 \times 10^{-4}$ & $-5.93 \times 10^{-5}$ & $5.72 \times 10^{-4}$ & 0.01 & 2.42 & 0.10 & 2.82 \\
\hline $\mathrm{Ni} \mathrm{I}$ & 676.78 & 0.66 & 4.32 & 0.90 & $+2.18 \times 10^{-4}$ & $-1.11 \times 10^{-3}$ & $5.55 \times 10^{-4}$ & 0.10 & 2.57 & 0.65 & 4.36 \\
\hline $\mathrm{Fe}$ I & 709.04 & 0.47 & 3.87 & 0.90 & $+6.79 \times 10^{-5}$ & $-8.43 \times 10^{-4}$ & $5.38 \times 10^{-4}$ & 0 & 0 & 0 & 0 \\
\hline $\mathrm{Fe}$ II & 722.45 & 0.12 & 3.43 & 0.91 & $+2.87 \times 10^{-4}$ & $-2.28 \times 10^{-4}$ & $5.30 \times 10^{-4}$ & 0 & 0 & 0 & 0 \\
\hline Si I & 1062.76 & 0.39 & 10.60 & 0.92 & $+2.62 \times 10^{-4}$ & $-4.36 \times 10^{-3}$ & $4.21 \times 10^{-4}$ & 0.07 & 7.27 & 0.39 & 11.03 \\
\hline $\mathrm{Fe} I$ & 1089.63 & 0.31 & 5.25 & 0.93 & $-1.56 \times 10^{-4}$ & $-8.98 \times 10^{-4}$ & $3.92 \times 10^{-4}$ & 0.07 & 4.72 & 0.29 & 5.52 \\
\hline $\mathrm{Fe} \mathrm{I}$ & 1142.23 & 0.51 & 7.13 & 0.93 & $-6.35 \times 10^{-6}$ & $-2.49 \times 10^{-3}$ & $3.78 \times 10^{-4}$ & 0.14 & 5.73 & 0.48 & 7.53 \\
\hline $\mathrm{Fe} \mathrm{I}$ & 1221.33 & 0.11 & 6.14 & 0.93 & $-3.06 \times 10^{-5}$ & $-1.61 \times 10^{-3}$ & $3.44 \times 10^{-4}$ & 0.02 & 5.98 & 0.10 & 7.37 \\
\hline $\mathrm{Fe} \mathrm{I}$ & 1558.83 & 0.26 & 11.07 & 0.91 & $+5.16 \times 10^{-5}$ & $-7.21 \times 10^{-3}$ & $2.25 \times 10^{-4}$ & 0.06 & 8.98 & 0.26 & 12. 01 \\
\hline $\mathrm{Fe} I$ & 1564.85 & 0.28 & 10.41 & 0.92 & $+4.04 \times 10^{-7}$ & $-6.22 \times 10^{-3}$ & $2.21 \times 10^{-4}$ & 0.06 & 9.58 & 0.22 & 13.67 \\
\hline
\end{tabular}


D. Cabrera Solana et al.: Sensitivity of weak spectral lines, Online Material p 4

Table A.3. Same as Table A.1, for the umbral model.

\begin{tabular}{|c|c|c|c|c|c|c|c|c|c|c|c|}
\hline Species & $\lambda_{0}[\mathrm{~nm}]$ & $A_{0}$ & $A_{1}[\mathrm{pm}]$ & $A_{2}$ & $\partial A_{0} / \partial T\left[\mathrm{~K}^{-1}\right]$ & $\partial A_{1} / \partial T[\mathrm{pm} / \mathrm{K}]$ & $\partial A_{2} / \partial T\left[\mathrm{~K}^{-1}\right]$ & $A_{0}^{V}$ & $A_{1}^{V}[\mathrm{pm}]$ & $A_{0}^{*}$ & $A_{1}^{*}[\mathrm{pm}]$ \\
\hline Ni I & 491.20 & 0.18 & 2.90 & 0.30 & $+3.02 \times 10^{-4}$ & $-7.37 \times 10^{-4}$ & $3.24 \times 10^{-4}$ & 0 & 0 & 0 & 0 \\
\hline $\mathrm{Fe} \mathrm{I}$ & 524.71 & 0.33 & 4.53 & 0.32 & $+3.73 \times 10^{-4}$ & $-3.26 \times 10^{-3}$ & $3.34 \times 10^{-4}$ & 0.15 & 3.56 & 0.33 & 4.56 \\
\hline $\mathrm{Fe} I$ & 525.02 & 0.33 & 4.46 & 0.32 & $+3.71 \times 10^{-4}$ & $-3.09 \times 10^{-3}$ & $3.34 \times 10^{-4}$ & 0.16 & 3.96 & 0.33 & 4.53 \\
\hline $\mathrm{Fe} I$ & 537.96 & 0.25 & 3.64 & 0.33 & $+3.28 \times 10^{-4}$ & $-1.22 \times 10^{-3}$ & $3.34 \times 10^{-4}$ & 0.09 & 2.55 & 0.25 & 3.65 \\
\hline Ti II & 538.10 & 0.16 & 2.94 & 0.33 & $+3.44 \times 10^{-4}$ & $-2.38 \times 10^{-4}$ & $3.33 \times 10^{-4}$ & 0.07 & 2.21 & 0.16 & 2.95 \\
\hline $\mathrm{Fe} I$ & 557.61 & 0.26 & 10.31 & 0.33 & $+3.15 \times 10^{-4}$ & $-1.07 \times 10^{-2}$ & $3.37 \times 10^{-4}$ & 0 & 0 & 0 & 0 \\
\hline $\mathrm{Fe} \mathrm{I}$ & 569.15 & 0.19 & 3.69 & 0.34 & $+3.13 \times 10^{-4}$ & $-1.66 \times 10^{-3}$ & $3.39 \times 10^{-4}$ & 0 & 0 & 0 & 0 \\
\hline $\mathrm{Fe}$ II & 614.93 & 0.04 & 3.45 & 0.36 & $+1.65 \times 10^{-4}$ & $-9.09 \times 10^{-4}$ & $3.43 \times 10^{-4}$ & 0.02 & 2.96 & 0.04 & 3.49 \\
\hline $\mathrm{Fe} \mathrm{I}$ & 617.33 & 0.31 & 4.23 & 0.36 & $+3.37 \times 10^{-4}$ & $-1.20 \times 10^{-3}$ & $3.45 \times 10^{-4}$ & 0.15 & 3.98 & 0.31 & 4.32 \\
\hline $\mathrm{Fe} I$ & 630.25 & 0.27 & 6.66 & 0.37 & $+3.41 \times 10^{-4}$ & $-5.50 \times 10^{-3}$ & $3.45 \times 10^{-4}$ & 0.12 & 5.20 & 0.26 & 6.76 \\
\hline $\mathrm{Fe} I$ & 630.35 & 0.04 & 3.08 & 0.37 & $+8.68 \times 10^{-5}$ & $-1.36 \times 10^{-3}$ & $3.45 \times 10^{-4}$ & 0.02 & 2.89 & 0.04 & 3.14 \\
\hline Ti I & 630.38 & 0.29 & 3.29 & 0.37 & $+1.35 \times 10^{-4}$ & $-1.62 \times 10^{-3}$ & $3.46 \times 10^{-4}$ & 0.12 & 2.96 & 0.29 & 3.31 \\
\hline $\mathrm{Ni} \mathrm{I}$ & 676.78 & 0.34 & 5.15 & 0.39 & $+3.41 \times 10^{-4}$ & $-1.58 \times 10^{-3}$ & $3.50 \times 10^{-4}$ & 0.15 & 4.21 & 0.34 & 5.19 \\
\hline Fe I & 709.04 & 0.23 & 4.54 & 0.40 & $+3.21 \times 10^{-4}$ & $-1.43 \times 10^{-3}$ & $3.49 \times 10^{-4}$ & 0 & 0 & 0 & 0 \\
\hline $\mathrm{Fe}$ II & 722.45 & 0.01 & 3.88 & 0.41 & $+4.83 \times 10^{-5}$ & $-1.22 \times 10^{-3}$ & $3.48 \times 10^{-4}$ & 0 & 0 & 0 & 0 \\
\hline Si I & 1062.76 & 0.14 & 12.94 & 0.52 & $+2.94 \times 10^{-4}$ & $-9.31 \times 10^{-3}$ & $3.18 \times 10^{-4}$ & 0.06 & 10.84 & 0.13 & 13.06 \\
\hline $\mathrm{Fe} \mathrm{I}$ & 1089.63 & 0.25 & 6.13 & 0.53 & $+2.67 \times 10^{-4}$ & $-1.43 \times 10^{-3}$ & $3.20 \times 10^{-4}$ & 0.12 & 5.97 & 0.24 & 6.37 \\
\hline $\mathrm{Fe} I$ & 1142.23 & 0.38 & 9.47 & 0.54 & $+2.99 \times 10^{-4}$ & $-5.69 \times 10^{-3}$ & $3.18 \times 10^{-4}$ & 0.19 & 9.70 & 0.36 & 9.89 \\
\hline $\mathrm{Fe} \mathrm{I}$ & 1221.33 & 0.08 & 7.78 & 0.57 & $+1.89 \times 10^{-4}$ & $-7.02 \times 10^{-3}$ & $2.98 \times 10^{-4}$ & 0.04 & 7.61 & 0.07 & 8.89 \\
\hline $\mathrm{Fe} \mathrm{I}$ & 1558.83 & 0.21 & 17.75 & 0.70 & $+2.71 \times 10^{-4}$ & $-1.92 \times 10^{-2}$ & $2.00 \times 10^{-4}$ & 0.10 & 14.94 & 0.20 & 18.10 \\
\hline Fe I & 1564.85 & 0.24 & 17.14 & 0.70 & $+2.89 \times 10^{-4}$ & $-1.88 \times 10^{-2}$ & $1.98 \times 10^{-4}$ & 0.12 & 15.99 & 0.21 & 19.31 \\
\hline
\end{tabular}

